\title{
A Problem for Credal Consequentialism
}

\author{
Michael Caie*
}

Beliefs, we'll assume, come in degrees. As a short-hand, we'll refer to these graded doxastic attitudes as credences, and to the totality of an agent's credences as their credal state.

Plausibly, there are certain epistemically good-making features that an agent's credal state may have. Credences, for example, may be closer or further from the truth, and it is at least prima facie plausible that the closer an agent's credences are, on average, to the truth the better such credences are epistemically. ${ }^{1}$

Given an appropriately well-defined notion of the epistemic good for credal states, it is tempting to think that the facts concerning which credal states are rationally permissible or obligatory for an agent may be grounded in facts about the extent to which such credal states appear, by the lights of the agent, to be conducive to the attainment of the epistemic good. $^{2}$ Call this sort of view credal consequentialism. For example, it is tempting to think that an agent ought rationally to adopt those credences that, by her lights, would be most conducive to the attainment of the epistemic good. Credal rationality, on this picture, consists in maximizing the expected epistemic utility of one's credal state.

Like consequentialist accounts in ethics, credal consequentialism has the virtue of being simple and principled. However, like consequentialist accounts in ethics, credal consequentialism has some surprising consequences that are at odds with our pre-theoretic judgments. First, given plausible accounts of the epistemic good for credal states, this account councils the acceptance of so-called epistemic bribes; in certain cases, by adopting apparently irrational credences in particular propositions an agent may ensure that their other credences have certain epistemic good-making features, and so, in certain cases, an agent may maximize their overall epistemic utility by adopting credences in particular propositions that seem irrational. ${ }^{3}$ Second, given plausible accounts of the epistemic good for credal states,

${ }^{*}$ Forthcoming in Epistemic Consequentialism, Jeffrey Dunn and Kristoffer Ahlstrom-Vij (eds.), OUP. Thanks to the editors and anonymous referees of this volume. Special thanks to Jason Konek and Cian Dorr for very helpful comments on a draft of this paper.

${ }^{1}$ See Joyce (1998) and Joyce (2009) for an assessment of different ways of developing the idea that the principal good-making feature for credal states consists in their closeness to the truth. For alternative proposals according to which the principal good-making feature of credal states consists in their closeness to chances see Hájek (n.d.), Pettigrew (2012), and Caie (2015).

${ }^{2}$ See, however, Konek and Levinstein (forthcoming) for an account that attempts to ground facts about credal rationality in facts about the epistemic good for credal states in a distinctively non-consequentialist manner.

${ }^{3}$ For arguments to this effect see, for example, Jenkins (2007), Greaves (2013), and Berker (2013). 
this account entails that in certain cases an agent may be rationally required to have probabilistically incoherent credences. ${ }^{4}$ And, finally, given plausible accounts of the epistemic good for credal states, this account entails that in certain cases a rational agent should update their credences in a manner that violates conditionalization and its natural generalizations. ${ }^{5}$

Some may take these consequences to provide decisive refutation of credal consequentialism. I'm less certain. In the ethical case, it seems to me that, given the simplicity and principled character of consequentialist theories, the fact that such theories appear to provide the right judgments in a large range of central cases makes it a delicate question-one without any obvious answer - whether those cases in which consequentialist theories are at odds with our pre-theoretic judgments should be seen as counterexamples or as surprising discoveries. Similarly, I think that if credal consequentialism is able to provide us with an account that delivers apparently reasonable judgments in a sufficiently large number of central cases, then it becomes a delicate question whether epistemic rationality may in fact sanction epistemic bribes, demand probabilistic incoherence or violations of updating by conditionalization.

In what follows, though, I'll argue that credal consequentialism is at odds with our pre-theoretic judgments in a much wider class of cases than has so far be acknowledged. In particular, I'll show that there are a great number of cases in which, intuitively, there may be a non-trivial distinction between credences that are rationally permissible and those that are impermissible, but that in such cases credal consequentialism either entails that every possible credal state is rationally permissible, or that every possible credal state is rationally impermissible, or that credal states, in such cases, cannot be assessed as rationally permissible or impermissible. The picture of credal rationality that emerges from credal consequentialism, then, is one that would seem to bear little resemblance to our pre-theoretic picture.

\section{Causal Decision Theory}

The guiding thought behind credal consequentialism is that epistemic rationality requires that an agent adopt those credences that, by her lights, would be most conducive to the attainment of the epistemic good. This intuitive idea can be sharpened up with the tools of causal decision theory.

In this section, I'll outline some of the basic elements of causal decision theory. In $§ 1.1$, I'll provide a characterization of causal expectation values. In $\S 1.2$, I'll show how such expectation values may be used to yield deontic verdicts concerning the possible options available to an agent. In particular, I'll argue that any adequate version of causal decision theory will entail, given certain cases in which an agent lacks well-defined expectation values for the utility of certain options available to them, either that every option available to them is rationally permissible, or that every option available to them is rationally impermissible, or that every option available to them is not apt for rational assessment.

\footnotetext{
${ }^{4}$ For cases of this sort see Caie (2013).

${ }^{5}$ For cases of this sort see Carr (n.d.).
} 
In $\S 2$, I'll show how the machinery of causal decision theory, outlined in this section, may be used to provide a precise characterization of credal consequentialism. I'll then show that in a large class of cases agents will lack well-defined expectation values for the credal options available to them in such a manner that, given credal consequentialism, it either follows that every credal option available to them is rationally permissible, or that every credal option available to them is rationally impermissible, or that every credal option available to them is not apt for rational assessment.

There are a number of different ways of developing the core ideas behind causal decision theory. In what follows, I'll focus on a version of causal decision theory inspired by, though in certain details distinct from, versions presented in Gibbard and Harper (1978) and Lewis (1981). While some of the details of the arguments that follow will depend on this particular choice, all the main points that I'll make could be made equally, mutatis mutandis, given alternative formulations.

\subsection{Causal Expectation Values}

Let $W$ be the set of epistemically possible worlds, i.e., ways that the world might be that cannot be ruled out a priori. We say that a set $P \subseteq W$ is a proposition. We say that a set of propositions $\mathbf{A}$ is a $\sigma$-algebra just in case $\mathbf{A}$ is closed under complementation and finite and countable unions. ${ }^{6}$ And we say that a $\sigma$-algebra $\mathbf{A}$ is atomic just in case $\mathbf{A}$ is such that there exists some At $\subset \mathbf{A}$ such that (i) each $P \in \mathbf{A t}$ is non-empty, (ii) At partitions $W$ and (iii) for each $P \in \mathbf{A t}$, there is no non-empty set $Q \in \mathbf{A}$ such that $Q \subset P{ }^{7}$ We'll say that the members of $\mathbf{A t}$ are the atoms of $\mathbf{A}$.

Let $\mathbf{A}_{i}^{t}$ be an atomic $\sigma$-algebra of propositions and let $\operatorname{Cr}_{i}^{t}(\cdot): \mathbf{A}_{i}^{t} \rightarrow \mathbb{R}$ be a function mapping members of $\mathbf{A}_{i}^{t}$ to real numbers. We take $C r_{i}^{t}(\cdot)$ to represent an agent $i$ 's credences at some time $t$. The members of $\mathbf{A}_{i}^{t}$ are those propositions that $i$ is able to entertain at $t$, and $\operatorname{Cr}_{i}^{t}(\cdot)$ represents how likely $i$ at $t$ thinks it is that each such proposition is true. ${ }^{8}$ We let $\mathbf{A t}_{i}^{t} \subset \mathbf{A}_{i}^{t}$ be the set of atoms of $\mathbf{A}_{i}^{t}$.

Let $u_{i}^{t}(\cdot): W \rightarrow \mathbb{R}$ be a function mapping members of $W$ to real numbers. We take $u_{i}^{t}(\cdot)$ to represent $i$ 's values at $t$. We have, then, that $u_{i}^{t}(w) \geq u_{i}^{t}\left(w^{\prime}\right)$ just in case, given $i$ 's values at $t, w$ is at least as desirable as $w^{\prime}{ }^{9}$

Let $\mathbf{O}_{i}^{t^{\prime}}$ be a set of propositions that partitions $W$. We assume that, for each $O \in \mathbf{O}_{i}^{t^{\prime}}$, it

\footnotetext{
${ }^{6}$ That is: if $P \in \mathbf{A}$, then $W-P \in \mathbf{A}$, and if $I$ is a finite or countable set of integers and $P_{i} \in \mathbf{A}$, for each $i \in I$, then $\cup\left\{P_{i}: i \in I\right\} \in \mathbf{A}$.

${ }^{7}$ We say that a set of propositions $\mathbf{P}$ partitions $\mathrm{W}$ just in case $\cup \mathbf{P}=W$ and, for each $P, Q \in \mathbf{P}, P \cap Q=\emptyset$.

${ }^{8}$ Note that all of the main points that follow could be made equally well if, instead of taking the objects of credence to be propositions, we took them to be centered-propositions, i.e., sets of world, time, individual triples. Such objects are appropriate, given agents who have essentially self-locating credences. See, for example, Lewis (1979b). For simplicity, however, we'll ignore this possible complication.

${ }^{9}$ Note that, if we like, we can take $u_{i}^{t}(\cdot): W \rightarrow \mathbb{R}$ to be derivative from a function $v_{i}^{t}(\cdot): \mathbf{A t}_{i}^{t} \rightarrow \mathbb{R}$. In particular, given such a $v_{i}^{t}(\cdot)$ we can say that, for each $P \in \mathbf{A} \mathbf{t}_{i}^{t}$ and each $w \in P, u_{i}^{t}(w)=v_{i}^{t}(P)$. Thus, even if an agent's conceptual resources don't allow her to distinguish $w$ from other worlds in $P$ we can still talk about the value of the world for that agent. Note, though, that we cannot do the same for $C r_{i}^{t}(\cdot)$ if we want to allow that such functions may be measures and so additive.
} 
is within $i$ 's power at $t^{\prime}$ to realize $O$ and that, for each $O \in \mathbf{O}_{i}^{t^{\prime}}$, there is no $O^{\prime} \subset O$ such that it is within $i$ 's power at $t^{\prime}$ to realize $O^{\prime}$. The members of $\mathbf{O}_{i}^{t^{\prime}}$, then, constitute the strongest propositions that $i$ is able to make true at $t^{\prime}$. We'll refer to this set as $i$ 's options at $t^{\prime}$.

Let $P$ be a set such that, for some $x \in \mathbb{R}, P=\left\{w \in W: u_{i}^{t}(w)=x\right\}$. We'll call such a set a t-value proposition, and we'll denote such a proposition by $\left[V_{i}^{t}=x\right]$, and the class of such propositions by $\mathbf{V}_{i}^{t}$.

Let $P \square \leftrightarrow Q$ be the non-backtracking counterfactual proposition that says that were $P$ the case, $Q$ would be the case. ${ }^{10}$ Given some $O \in \mathbf{O}_{i}^{t^{\prime}}$ and some $\left[V_{i}^{t}=x\right] \in \mathbf{V}_{i}^{t}, O \square \leftrightarrow\left[V_{i}^{t}=x\right]$, then, is the set of worlds $w$ such that, at $w$, were $i$ to realize $O$ at $t^{\prime}$, then the result would be a situation of utility $x$, given $i$ 's values at $t$.

Consider, then, a set that contains, for each $O \in \mathbf{O} \subseteq \mathbf{O}_{i}^{t^{\prime}}$, a unique proposition of the form $O \square \rightarrow\left[V_{i}^{t}=x\right]$, and no other propositions. We'll say that a non-empty intersection of such a set is a t-value dependence hypothesis for $\mathrm{O}$. A t-value dependence hypothesis for $\mathbf{O}$, then, tells us, for each $O \in \mathbf{O}$, how matters that $i$ values at $t$ depend counterfactually on $i$ realizing $O$ at $t^{\prime}$. We'll denote the set of t-value dependence hypotheses for $\mathbf{O} \subseteq \mathbf{O}_{i}^{t^{\prime}}$, $\mathbf{D}\left(\mathbf{O}, u_{i}^{t}\right)$. We'll assume that, in general, this set partitions $W{ }^{11}$

For each $O \in \mathbf{O}_{i}^{t^{\prime}}$ and each $D \in \mathbf{D}\left(\mathbf{O}_{i}^{t^{\prime}}, u_{i}^{t}\right)$, there will be a unique $x \in \mathbb{R}$ such that $\left(O \square \mapsto\left[V_{i}^{t}=x\right]\right) \cap D \neq \emptyset$. We let $U(O \cap D)$ be this unique $x$.

We want to provide a characterization of the extent to which $i$ at $t$ expects that good results would be brought about were they to realize $O$ at $t^{\prime}$. A standard way of doing this assumes that $\mathbf{D}\left(\mathbf{O}_{i}^{t^{\prime}}, u_{i}^{t}\right) \subseteq \mathbf{A}_{i}^{t}$ and then takes an agent $i$ 's expectation value at $t$ of the causal utility of realizing an option $O$ at $t^{\prime}$ to be given by: ${ }^{12}$

\footnotetext{
${ }^{10}$ Roughly speaking, a non-backtracking counterfactual $P \square \rightarrow Q$ is one that evaluates whether $Q$ would have been true, had $P$ been true, holding fixed as much of the past as is compatible with the truth of $P$. See Lewis (1973) and Lewis (1979a) for the distinction between backtracking and non-backtracking counterfactuals.

${ }^{11}$ This, it should be stressed, is a controversial assumption. Given plausible principles governing $\square \rightarrow$ this will follow if we assume, in addition, the principle of Conditional Excluded Middle (CEM): $(\phi \square \rightarrow$ $\psi) \vee(\phi \square \rightarrow \neg \psi)$. However, (CEM) is a notoriously controversial principle. David Lewis famously rejected it, while Robert Stalnaker endorsed it. (See Lewis (1973), Stalnaker (1981b) and Stalnaker (1981a) for some of the early controversy.) And those cases that have lead some to reject (CEM), have also lead people to reject the claim that, in general, the t-value dependence hypotheses for $\mathbf{O} \subseteq \mathbf{O}_{i}^{t^{\prime}}$ form a partition of $W$. (For arguments to this effect see Joyce (1999).) For what it's worth, I'm inclined to accept (CEM). (For some recent defenses of this principle see Williams (2010) and Goodman (n.d.).) If, however, one rejects $(\mathrm{CEM})$, and so too the assumption that the t-value dependence hypotheses for $\mathbf{O} \subseteq \mathbf{O}_{i}^{t^{\prime}}$ form a partition of $W$, then one will want to endorse an alternative formulation of causal decision theory. (See, for example, Joyce (1999).) However, let me note again that the main points that follow won't essentially depend upon this particular formulation of causal decision theory, and so won't depend essentially on the controversial assumption that t-value dependence hypotheses for $\mathbf{O} \subseteq \mathbf{O}_{i}^{t^{\prime}}$ form a partition of $W$.

${ }^{12}$ If the set of dependence hypotheses, $\mathbf{D}\left(\mathbf{O}_{i}^{t^{\prime}}, u_{i}^{t}\right)$, is uncountable, then we have instead:

$$
E C U_{i}^{t}(O)=\int_{D \in \mathbf{D}\left(\mathbf{O}_{i}^{t^{\prime}}, u_{i}^{t}\right)} U(O \cap D) d C r_{i}^{t}(D)
$$

Further qualifications of this sort will be required at various points throughout. To avoid tedium, though,
} 


$$
\text { ECU (Preliminary 1): } E C U_{i}^{t}(O)=\sum_{D \in \mathbf{D}\left(\mathbf{O}_{i}^{t^{\prime}}, u_{i}^{t}\right)} C r_{i}^{t}(D) U(O \cap D)
$$

This characterization of expected causal utility is perfectly fine, given the assumption that $\mathbf{D}\left(\mathbf{O}_{i}^{t^{\prime}}, u_{i}^{t}\right) \subseteq \mathbf{A}_{i}^{t}$. However, even if an agent isn't able to entertain each proposition in $\mathbf{D}\left(\mathbf{O}_{i}^{t^{\prime}}, u_{i}^{t}\right)$, they may still have a well-defined causal expectation value for some $O \in \mathbf{O}_{i}^{t^{\prime}}$. For while an agent may not be able to consider, for each $O \in \mathbf{O}_{i}^{t^{\prime}}$, each of the different ways in which outcomes may depend upon the realization of that option, it may nonetheless be that the agent is able to consider such dependencies for some $O \in \mathbf{O}_{i}^{t^{\prime}}$.

To see this, consider some arbitrary $O \in \mathbf{O}_{i}^{t^{\prime}}$. Given such an $O$, we can coarse-grain $\mathbf{D}\left(\mathbf{O}_{i}^{t^{\prime}}, u_{i}^{t}\right)$ by taking unions of the members of $\mathbf{D}\left(\mathbf{O}_{i}^{t^{\prime}}, u_{i}^{t}\right)$ that agree about what utility would result were $O$ to be realized. If an agent has credences defined over an algebra containing such a coarse-graining of $\mathbf{D}\left(\mathbf{O}_{i}^{t^{\prime}}, u_{i}^{t}\right)$, then the agent should be taken to have a well-defined expectation for the causal utility of $O$, even if the agent does not have a well-defined expectation value for the causal utility of each member of $\mathbf{O}_{i}^{t^{\prime}}$.

More generally, it may be that, while it's not the case that $\mathbf{D}\left(\mathbf{O}_{i}^{t^{\prime}}, u_{i}^{t}\right) \subseteq \mathbf{A}_{i}^{t}$, there is some $\mathbf{O} \subset \mathbf{O}_{i}^{t^{\prime}}$, such that $\mathbf{D}\left(\mathbf{O}, u_{i}^{t}\right) \subseteq \mathbf{A}_{i}^{t}{ }^{13}$ In this case, the agent should be taken to have a well-defined expectation value for the causal utility for each $O \in \mathbf{O}$. ECU (Preliminary 1), though, is silent about such cases. We need, then, a more general characterization of causal utility expectation values.

To this end, we can extend our characterization of $U(\cdot)$. In particular, let us say, for each $O \in \mathbf{O}_{i}^{t^{\prime}}$ and each $P \subseteq \mathbf{A}_{i}^{t}$, that $U(O \cap P)=x$ if $\left(O \square \rightarrow\left[V_{i}^{t}=x\right]\right) \cap P=P$, and otherwise $U(O \cap P)$ is undefined. $U(O \cap P)$, then, is well-defined just in case each world in $P$ agrees about what utility would result were $O$ to be realized. And, if $U(O \cap P)$ is well-defined, then this value is exactly the utility value that each member of $P$ agrees would result were $O$ to be realized.

Given this characterization of $U(\cdot)$, we can defined the following quantity:

$$
\text { Def. } G_{i}^{t}(O)={ }_{d f} \sum_{A \in \mathbf{A t}_{i}^{t}} C r_{i}^{t}(A) U(O \cap A)
$$

A natural way to try to address the limitation of our preceding characterization of expected causal utility, is to take the expected causal utility of an option $O$ for an agent $i$ at $t$ to be given by $G_{i}^{t}(O)$. Thus:

\section{ECU (Preliminary 2): $E C U_{i}^{t}(O)=G_{i}^{t}(O)$}

Unlike ECU (Preliminary 1), this allows that an agent may have well-defined causal utility expectation values for some, though perhaps not all, $O \in \mathbf{O}_{i}^{t^{\prime}}$. This may, for example, be

from now on I will, for the most part, leave such qualifications implicit.

${ }^{13}$ In typical cases, if $\mathbf{O} \subset \mathbf{O}_{i}^{t^{\prime}}$, then $\mathbf{D}\left(\mathbf{O}, u_{i}^{t}\right)$ will be a coarse-graining of $\mathbf{D}\left(\mathbf{O}_{i}^{t^{\prime}}, u_{i}^{t}\right)$. And so, an agent may have credences defined over an algebra that contains the former though not the latter. 
true if, for some $\mathbf{O} \subset \mathbf{O}_{i}^{t^{\prime}}, \mathbf{A t}_{i}^{t}=\mathbf{D}\left(\mathbf{O}, u_{i}^{t}\right)$. It may also be true if, for some $\mathbf{O} \subset \mathbf{O}_{i}^{t^{\prime}}, \mathbf{A t}_{i}^{t}$ is a fine-graining of $\mathbf{D}\left(\mathbf{O}, u_{i}^{t}\right)$ which is not equal to $\mathbf{D}\left(\mathbf{O}_{i}^{t^{\prime}}, u_{i}^{t}\right)$.

This characterization, though, is also too restrictive. For, given ECU (Preliminary 2), $E C U_{i}^{t}(O)$ will be undefined as long as there is some $A \in \mathbf{A t}_{i}^{t}$ and some $x \neq y$, such that, for some $w \in A, O \square \leftrightarrow\left[V_{i}^{t}=x\right]$ obtains at $w$, and, for some $w^{\prime} \in A, O \square \leftrightarrow\left[V_{i}^{t}=y\right]$ obtains at $w^{\prime}$. For, given such an $A \in \mathbf{A t}_{i}^{t}, U(O \cap A)$ will be undefined. It seems to me, though, that even if there are some $A \in \mathbf{A t}_{i}^{t}$ that are not homogeneous with respect to which utility would result were $O$ to be realized, if the agent's credal state rules out each such atom, then the agent should be taken to have a well-defined expectation value for the causal utility of $O$.

For example, suppose that an agent is certain that were $O$ to be realized, then a certain utility $x$ would result. In this case, it is clear that the agent has a well-defined expectation value for the casual utility of $O$, viz., $x$. But it's compatible with an agent being certain that were $O$ to be realized, then a certain utility $x$ would result, that there be certain ways in which utility may depend upon the realization of $O$ that the agent is not able to consider, and so some $A \in \mathbf{A t}_{t}^{t^{\prime}}$ that are not homogeneous about which utility would result were $O$ to be realized.

In light of this, we will take the expected causal utility, for $i$ at $t$, of an option $O \in \mathbf{O}_{i}^{t^{\prime}}$ to be characterized as follows. Let $\mathbf{Z}_{C r_{i}^{t}}=\left\{A \in \mathbf{A t}_{i}^{t}: C r_{i}^{t}(A) \neq 0\right\} .{ }^{14}$ Then we say:

$$
\text { ECU: } E C U_{i}^{t}(O)=\sum_{A \in \mathbf{Z}_{C r_{i}^{t}}} C r_{i}^{t}(A) U(O \cap A)
$$

It is worth pausing to comment on how we should think about those cases in which $E C U_{i}^{t}(O)$ is well-defined but $G_{i}^{t}(O)$ is not.

Let us say that an agent with credences $\operatorname{Cr}_{i}^{t}(\cdot)$ is quasi-opinionated about $\mathbf{D}\left(\{O\}, u_{i}^{t}\right)$ just in case there is some $\mathbf{D} \subset \mathbf{D}\left(\{O\}, u_{i}^{t}\right)$ such that $\cup \mathbf{Z}_{C r_{i}^{t}} \subseteq \cup \mathbf{D}$. If an agent is quasiopinionated about $\mathbf{D}\left(\{O\}, u_{i}^{t}\right)$ then they are certain that the utility value that would obtain were $O$ to be realized is amongst some proper subset of the set of a priori epistemically possible utility values that could be realized were $O$ to obtain.

Now, if $G_{i}^{t}(O)$ is not well-defined but $E C U_{i}^{t}(O)$ is, then $C r_{i}^{t}(\cdot)$ must be quasi-opinionated about $\mathbf{D}\left(\{O\}, u_{i}^{t}\right)$. In such cases, the agent's conceptual resources do not allow them to entertain all of the different ways in which $O$ might result in outcomes of differing utility. However, despite not being able to entertain all such dependencies, the agent is able to entertain some such dependencies, and, moreover, the agent lumps all of their credence on some subclass of $\mathbf{A t}_{t}^{t^{\prime}}$ that serve to characterize such dependencies. By being opinionated and ruling out certain dependence hypotheses for $O$, an agent may have a well-defined expectation value for $O$, even if the agent cannot entertain all of the ways in which utility may result were $O$ to be realized.

A consequence of this is that if $i$ is not quasi-opinionated about $\mathbf{D}\left(\{O\}, u_{i}^{t}\right)$, and there are some dependence hypotheses for $O$ that $i$ cannot entertain, then $i$ cannot have a well-defined

\footnotetext{
${ }^{14}$ Note that if $\mathbf{A t}_{i}^{t}$ is uncountable, then in the above characterization we should take $C r_{i}^{t}(\cdot)$ to be a density function.
} 
expectation value for the causal utility of $O$. This fact will play an important role in what follows.

\subsection{From Expectations to Deontic Verdicts}

According to causal decision theory, if there are options that maximize an agent's expected causal utility, then the agent ought to realize one of those options. As it stands, though, this claim is ambiguous. For the expected causal utility of an option depends on an agent's utilities and credences. But an agent's utilities and credences are time-relative. We need, then, a more precise characterization of how the deontic statuses of an agent's options at some time $t^{\prime}$ may be determined by the causal expectation values of those options.

To help avoid inessential and potentially distracting complications, in what follows, we'll impose a simplifying restriction on the class of decision problems with which we'll be concerned. We'll assume that, in a given decision problem, an agent has stable credences and utilities throughout some temporal interval leading up to $t^{\prime}$. Thus, we assume that, in a given decision problem, there is some $t^{\prime \prime}<t^{\prime}$, such that, for each $t_{1}, t_{2} \in\left(t^{\prime \prime}, t^{\prime}\right), \operatorname{Cr}_{i}^{t_{1}}(\cdot)=C r_{i}^{t_{2}}(\cdot)$ and $u_{i}^{t_{1}}(\cdot)=u_{i}^{t_{2}}(\cdot)$. For present purposes, this assumption involves no important loss of generality.

Given this, let us define the following, perhaps partial, function from $\mathbf{O}_{i}^{t^{\prime}}$ to $\mathbb{R}$ :

Def. $E C U_{i}(O)=x$ just in case, for all $t \in\left(t^{\prime \prime}, t^{\prime}\right), E C U_{i}^{t}(O)=x$

Note that, given our simplifying assumption, $\operatorname{ECU}_{i}(O)$ will be well-defined as long at $E C U_{i}^{t}(O)$ is well-defined for some $t \in\left(t^{\prime \prime}, t^{\prime}\right)$.

We can now provide the following precisification of the guiding idea behind causal decision theory:

Maximization: Let $M$ be the set of $O \in \mathbf{O}_{i}^{t^{\prime}}$ such that, for each $O^{\prime} \in \mathbf{O}_{i}^{t^{\prime}}$, $E C U_{i}(O) \geq E C U_{i}\left(O^{\prime}\right)$. If $M$ is non-empty, then, for each $O \in M$, it is rationally permissible for $i$ to realize $O$, and it is rationally required for $i$ to realize some $O \in M .^{15}$

${ }^{15}$ The natural generalization of Maximization is:

Diachronic Maximization: Let $M$ be the set of $O \in \mathbf{O}_{i}^{t^{\prime}}$ such that, for some $t^{\prime \prime}<t^{\prime}, \operatorname{ECU}_{i}^{t}(O) \geq$ $E C U_{i}^{t}\left(O^{\prime}\right)$, for every $O^{\prime} \in \mathbf{O}_{i}^{t^{\prime}}$ and every $t \in\left(t^{\prime \prime}, t^{\prime}\right)$. If $M$ is non-empty, then, for each $O \in M$, it is rationally permissible for $i$ to realize $O$, and it is rationally required for $i$ to realize some $O \in M$.

An alternative way of precisifying the core idea behind causal decision theory is:

Synchronic Maximization: Let $M$ be the set of $O \in \mathbf{O}_{i}^{t^{\prime}}$ such that, for each $O^{\prime} \in \mathbf{O}_{i}^{t^{\prime}}$, $E C U_{i}^{t^{\prime}}(O) \geq E C U_{i}^{t^{\prime}}\left(O^{\prime}\right)$. If $M$ is non-empty, then, for each $O \in M$, it is rationally permissible for $i$ to realize $O$, and it is rationally required for $i$ to realize some $O \in M$.

I'm inclined to think that Diachronic Maximization, and its special case Maximization, provide a better account than Synchronic Maximization of how the deontic status of an agent's options at $t^{\prime}$ may be determined by the agent's expectations of causal utility. In support of this, let me note two worries for Synchronic 
Maximization tells us (for an agent with stable credences and utilities leading up to $t^{\prime}$ ) which options are rationally permissible and which rationally impermissible at $t^{\prime}$, given that there is a non-empty set of options $M$ that maximize expected causal utility. This, however, leaves open the deontic status of such an agent's t'-options in those cases in which the set of options that maximize expected causal utility is empty. There are two important classes of such cases.

One way in which this type of case may arise is as follows. Given a credal state $C r_{i}^{t}(\cdot)$ and utility function $u_{i}^{t}(\cdot)$, it may be that, for each option $O$, there is some option $O^{\prime}$, such that $E C U_{i}^{t}\left(O^{\prime}\right)>E C U_{i}^{t}(O) .{ }^{16}$ And so it may be that, given an agent with stable credences and utilities throughout some interval leading up to $t^{\prime}$, for each option $O$, there is some option $O^{\prime}$, such that $E C U_{i}\left(O^{\prime}\right)>E C U_{i}(O)$.

Another way in which this type of case may arise is if, given the agent's stable credences and utilities leading up to $t^{\prime}$, there are no well-defined causal expectation values for at least some of the members of $\mathbf{O}_{i}^{t^{\prime}}$.

In what follows, our main concern will be with what we should say concerning certain cases of the second sort. It will help us, however, in addressing this question to first consider what we should say about the first type of case. There are, I think, three principled options for treating such cases, and, between these three options, one seems to me to be clearly

Maximization. First, it is natural to think that the credences and utilities of an agent that determine the expectation values that rationalize certain options for the agent should, at least sometimes, also be the things that cause the agent to realize some option that is so rationalized. However, it is hard to see how this could be so if the expectation values that rationalize an agent's options are determined by the credences and utilities that they have at the time that the option is realized.

Second, there is good reason to think that Synchronic Maximization is incompatible with a plausible oughtimplies-can principle. For there are cases in which, given a set of options, each option $O$ is such that if the agent learns that she has realized $O$, then, given this information, expected causal utility will be maximized by some option other than $O$. (For a case with this structure see Death in Damascus in Gibbard and Harper (1978).) But consider, then, an agent who is perfectly introspective about their own choices, so that it's guaranteed that at $t$ they are aware of their choice at $t$. Such an agent will be guaranteed to realise an option at $t$ that fails to maximize expected utility given their utilities and credences at $t$. Given Synchronic Maximization, then, such an agent will be guaranteed to be in violation of certain practical requirements of rationality. One might naturally think, though, that practical rationality should not preclude an agent from making a rational choice simply given that the agent manifests certain epistemic virtues, such as being aware of their own choices.

It's worth noting, though, that all of the main points that I'll make have natural analogues given the natural alternative version of causal decision theory that endorses Synchronic Maximization.

It's also worth noting that neither Synchronic Maximization nor Diachronic Maximization nor its special case Maximization are usually considered in presentations of decision theory. Instead, it's typically taken that there is some particular time of deliberation $t<t^{\prime}$ that is relevant to the deontic status of one's $t^{\prime}$ options. Taken literally, though, this seems to me completely implausible. A more charitable interpretation is that the assumption that there is some particular time prior to the time of choice that is specially relevant to the deontic status of one's later choices is simply a fiction used to simplify the decision theoretic models. Insofar, though, as one thinks that it is not the expected utilities at $t^{\prime}$ that are relevant to the deontic status of one's $t^{\prime}$-options, then it seems to me that Maximization, and more generally Diachronic Maximization, present a natural account of how the expected utilities of one's $t^{\prime}$-options at earlier times serve to determine deontic statuses for such options.

${ }^{16}$ For cases of this sort see Pollock (1983). 
preferable. The three principled ways of treating such cases are:

Global Dominance Permissivism: If, for each option $O$, there is some option $O^{\prime}$, such that $E C U_{i}\left(O^{\prime}\right)>E C U_{i}(O)$, then, for each $O \in \mathbf{O}_{i}^{t^{\prime}}$, it is rationally permissible for $i$ to realize $O$.

Global Dominance Prohibitionism: If, for each option $O$, there is some option $O^{\prime}$, such that $E C U_{i}\left(O^{\prime}\right)>E C U_{i}(O)$, then, for each $O \in \mathbf{O}_{i}^{t^{\prime}}$, it is rationally impermissible for $i$ to realize $O$.

Global Dominance Conservatism: If, for each option $O$, there is some option $O^{\prime}$, such that $E C U_{i}\left(O^{\prime}\right)>E C U_{i}(O)$, then, for each $O \in \mathbf{O}_{i}^{t^{\prime}}$, it is neither rationally permissible nor rationally impermissible for $i$ to realize $O$.

Granting that we should treat alike all cases in which, for each option that's available to an agent, there is another more attractive option, to reject each of these claims, one must maintain that, in such cases, some options are rationally permissible while others are impermissible. How might one distinguish, in such cases, between those options that are permissible and those that are impermissible?

One option would be to appeal to certain ordinal facts. For example, suppose that the ordering amongst an agent's options is isomorphic to the ordering amongst the natural numbers. Then one might maintain that there is some value $n$ such that if $O$ 's expected utility is better than less than $n$ other options then $O$ is rationally impermissible, but if $O$ 's expected utility is better than $n$ or more other options then $O$ is rationally permissible.

Another option would be to appeal to some threshold value for $E C U_{i}(\cdot)$, so that, for some $x, O$ is rationally permissible just in case $E C U_{i}(O) \geq x$.

The problem with these and other ways of trying to effect some non-trivial distinction between the class of permissible and impermissible options in such cases is that they seem completely unprincipled when combined with Maximization. And Maximization is extremely plausible.

Consider the threshold view. If there is some threshold for expected causal utility that suffices for an option to be rationally permissible in cases in which, for each option that's available to an agent, there is another more attractive option, then it becomes a mystery why there is not a similar threshold in cases in which there are some options that maximize expected utility. But it certainly seems that if there are some options that maximize expected utility then it is rationally impermissible to realize any other such option. Of course, one could simply maintain that, as a brute fact, there is threshold rationalization of options when, but only when, there are no maximal options. But this seems to me to be objectionably ad-hoc.

Similar considerations could be adduced against the claim that certain ordinal facts serve to effect a non-trivial distinction between the permissible and impermissible options in cases in which there are no options that maximize expected utility.

The more general point is that Maximization would seem to require that we assign the same deontic status to options that are alike with respect to the existence of some expected 
utility dominating alternative. If this principle is to be respected in those cases in which, for each option that's available to an agent, there is another more attractive option, then one must endorse either Global Dominance Permissivism, Global Dominance Prohibitionism, or Global Dominance Conservatism.

Which of these, though, should one endorse?

I don't have a compelling argument against Global Dominance Conservatism. Still, I think it should be considered the option of last resort. For while I'm perfectly happy to say that, in cases in which an agent doesn't have an appropriate view concerning the expected utility of their options, one cannot assess the realization of an option by the agent as rationally permissible or impermissible, it seems to me that rationality should not remain silent in cases in which an agent does have a view concerning the expected utility of all of their options. At the very least, if there is a sufficiently attractive option that allows for rational verdicts in such cases, then such an option should be preferred to Global Dominance Conservatism. And I think that there is a sufficiently attractive alternative.

Let's turn, then, to Global Dominance Prohibitionism. There is a pretty straightforward argument for this principle that is at least initially compelling. For, in the cases to which Maximization applies, the existence, for some option $O$, of an expected utility dominating alternative, entails that $O$ is rationally impermissible. And this principle, applied generally, entails Global Dominance Prohibitionism.

There is, however, a serious problem with Global Dominance Prohibitionism. For consider the following plausible principle:

Possible Permissibility: If, for each $O \in \mathbf{O}_{i}^{t^{\prime}}, E C U_{i}(O)$ is well-defined, and if $i$ is not guilty of any antecedent rational failing, then there must be some $O \in \mathbf{O}_{i}^{t^{\prime}}$ that is rationally permissible.

Now it seems to me completely implausible to maintain that an agent is irrational simply in virtue of having credences and utilities that entail that, for any option $O$, there is some other option $O^{\prime}$ such that $E C U_{i}\left(O^{\prime}\right)>E C U_{i}(O)$. Given this, it follows that Possible Permissibility is incompatible with Global Dominance Prohibitionism. Possible Permissibility, though, seems to me to be much more plausible than Global Dominance Prohibitionism. In light of this incompatibility, then, I think we should reject Global Dominance Prohibitionism.

Finally, let's consider Global Dominance Permissivism. We can also provide an argument for this principle by appeal to Maximization. To see this, note that both Maximization and Global Dominance Permissivism share the following natural property. In the cases in which they issues verdicts, each is the most conservative principle with respect to rational permissibility that is compatible with Possible Permissibility and that treats alike options that are alike with respect to the existence of some expected utility dominating alternative. We can see both of these principles, then, as being motivated by the following more general principle of rationality:

Permissible Dominance Symmetry: Given an agent with stable credences and utilities for some interval leading up to $t^{\prime}$ that determine a well-defined expected 
causal utility for each of their available $t^{\prime}$-options, there must be an available rational option, but only so many as are required in order not to draw deontic distinctions amongst cases that are alike with respect to the existence of dominating alternatives.

This strikes me as a plausible principle. The fact, then, that Global Dominance Permissivism follows from a plausible general principle from which Maximization also follows, gives us, I think, good reason to accept the former principle, in addition to the latter.

Let's now consider the second type of case in which Maximization leaves open the deontic status of an agent's options. In such cases, there are some $O \in \mathbf{O}_{i}^{t^{\prime}}$ for which $E C U_{i}(\cdot)$ is not well-defined. Given our purposes in this paper, we'll want to consider, more specifically, what we should say about those cases in which there are an infinite number of $O \in \mathbf{O}_{i}^{t^{\prime}}$ for which $E C U_{i}(\cdot)$ is not well-defined.

Let $\mathbf{O}^{U} \subseteq \mathbf{O}_{i}^{t^{\prime}}$ be the class of the agent's $t^{\prime}$-options for which $E C U_{i}(\cdot)$ is not well-defined. And let $\mathbf{O}^{D}={ }_{d f} \mathbf{O}_{i}^{t^{\prime}}-\mathbf{O}^{U}$, so that $\mathbf{O}^{D}$ is the class of the agent's $t^{\prime}$-options for which $E C U_{i}(\cdot)$ is well-defined. I claim:

No Distinctions: If $E C U_{i}(\cdot)$ is not well-defined for an infinite $\mathbf{O}^{U} \subseteq \mathbf{O}_{i}^{t^{\prime}}$, then, either, for each $O \in \mathbf{O}_{i}^{t^{\prime}}$, it is rationally permissible for $i$ to realize $O$, or, for each $O \in \mathbf{O}_{i}^{t^{\prime}}$, it is rationally impermissible for $i$ to realize $O$, or, for each $O \in \mathbf{O}_{i}^{t^{\prime}}$, it is neither rationally permissible nor rationally impermissible for $i$ to realize $O$.

In support of this, I'll argue that if there are non-trivial deontic distinctions amongst an agent's options in cases in which $\mathbf{O}^{U}$ is non-empty, then if $E C U_{i}(\cdot)$ is not well-defined for an infinite $\mathbf{O}^{U} \subseteq \mathbf{O}_{i}^{t^{\prime}}$, then, for each $O \in \mathbf{O}_{i}^{t^{\prime}}$, it is rationally permissible for $i$ to realize $O$. This claim entails No Distinctions and so the argument for the former serves as an argument for the latter.

Let us ask, then, how one might draw non-trivial deontic distinctions amongst an agent's options in cases in which $\mathbf{O}^{U}$ is non-empty? It seems clear that whatever we say about the deontic status of an agent's options in such a case, we should take each member of $\mathbf{O}^{U}$ to have the same deontic status. Given this constraint, here's a view one might find initially attractive:

Impermissible Undefinedness: For each $O \in \mathbf{O}^{U}$, it is rationally impermissible for $i$ to realize $O$. Let $\mathbf{O}^{M} \subseteq \mathbf{O}^{D}$ be the set of options that maximize expected causal utility amongst the members of $\mathbf{O}^{D}$. If $\mathbf{O}^{M} \neq \emptyset$, then it is rationally obligatory that one realize some option in $\mathbf{O}^{M}$. And if $\mathbf{O}^{M}=\emptyset$, then each $O \in \mathbf{O}^{D}$ is rationally permissible.

The guiding thoughts behind this principle are, first, that an agent ought not realize an option about which they have no view concerning its expected utility, and, second, that, having ruled out such options, we may then apply to the agent's remaining options those principles that apply in cases in which an agent has well-defined expectation values for all 
of the options that are available to them, treating the remaining options as the total set of options.

While this may seem at least prima facie plausible, it doesn't, I think, hold up under scrutiny. The problem with Impermissible Undefinedness is that, given Maximization and Global Dominance Permissivism, what would seem to matter for determining the permissibility or impermissibility of an agent's options is how attractive the options appear relative to one another. If, though, $E C U_{i}(\cdot)$ is not well-defined for some $O \in \mathbf{O}_{i}^{t^{\prime}}$, then, for every $O^{\prime} \in \mathbf{O}^{D}$, there will be no well-defined relation of relative attractiveness between $O$ and $O^{\prime}$. There is, then, a symmetry with respect to the well-definiedness of the relative attractiveness of any $O \in \mathbf{O}^{U}$ and any $O^{\prime} \in \mathbf{O}^{D}$. And, given this symmetry and the assumption that what matters for determining the permissibility or impermissibility of an agent's options is how attractive the options appear relative to one another, it is hard to see why each member of $\mathbf{O}^{U}$ should be ruled out as impermissible, while the same is not true of $\mathbf{O}^{D}$.

It seems to me, then, that a reasonable constraint on any principle that serves to draw non-trivial deontic distinctions amongst an agent's options in cases in which $\mathbf{O}^{U}$ is non-empty, is that such a principle respect these sorts of symmetries with respect to the well-definiedness of the relative attractiveness of an agent's options. Since Impermissible Undefinedness fails to satisfy this constraint, we have, I think good reason to reject this principle.

In cases in which $\mathbf{O}^{U}$ is empty, an agent's credences and utilities determine a complete ordering amongst the members of $\mathbf{O}_{i}^{t^{\prime}}$ that captures the relative attractiveness of these options by the light's of the agent. ${ }^{17}$ In cases, though, in which $\mathrm{O}^{U}$ is non-empty, while an agent's credences and utilities determine an ordering amongst the members of $\mathbf{O}^{D}$ that captures the relative attractiveness of these options by the light's of the agent, such credences and utilities leave open how attractive the members of $\mathbf{O}^{U}$ are both relative to one another and relative to the members of $\mathbf{O}^{D}$. In such cases, we can think of there being a non-empty non-singleton set of orderings amongst the agent's options that correspond to the different orderings capturing the relative attractiveness of the agent's options that are left open given their credences and utilities.

A principle that draws non-trivial deontic distinctions amongst an agent's options in cases in which $\mathbf{O}^{U}$ is non-empty, then, should tell us which options are rationally permissible and which rationally impermissible given such a set of orderings amongst the members of $\mathrm{O}_{i}^{t^{\prime}}$.

There are, furthermore, two natural constraints on such principles that I think we should endorse. First, in determining the deontic status of an option, I think that no ordering that is left open given the agent's credences and utilities should be privileged over any other. This, I take it, is part of what respecting the symmetries with respect to the well-definedness of the relative attractiveness of an agent's options involves. Second, I think we should take it that, given a set of orderings amongst the members of $\mathbf{O}_{i}^{t^{\prime}}$, the deontic status of some option $O$ should be a function of the deontic status that $O$ would have, relative to each ordering amongst the set, were that ordering to be the unique ordering given the agent's credences

\footnotetext{
${ }^{17}$ Strictly speaking these determine a complete pre-ordering. I'll, however, drop the prefix throughout this discussion.
} 
and utilities.

Given these two constraints, there are two principled ways that I can see of potentially drawing non-trivial deontic distinctions in cases in which $\mathbf{O}^{U}$ is non-empty, and, between these two options, one option seems to me to be clearly preferable.

We'll say that a function $E C U_{i}^{*}(\cdot): \mathbf{O}_{i}^{t^{\prime}} \rightarrow \mathbb{R}$ is an extension of $E C U_{i}(\cdot)$ just in case, for each $O \in \mathrm{O}^{D}, E C U_{i}^{*}(O)=E C U_{i}(O)$. Given, then, an agent $i$ with stable credences and utilities leading up to $t^{\prime}$, let $\mathcal{E}_{i}$ be the set of $E C U_{i}^{*}(\cdot): \mathbf{O}_{i}^{t^{\prime}} \rightarrow \mathbb{R}$ that extend the function $E C U_{i}(\cdot)$ defined by their stable credences and utilities. Consider, then, the following principles for determining the deontic status of the agent's $t^{\prime}$-options:

Deontic Supervaluationism: An option $O \in \mathbf{O}_{i}^{t^{\prime}}$ is permissible just in case it is permissible given each $E C U_{i}^{*}(\cdot) \in \mathcal{E}_{i}$.

Deontic Subvaluationism: An option $O \in \mathbf{O}_{i}^{t^{\prime}}$ is permissible just in case it is permissible given some $E C U_{i}^{*}(\cdot) \in \mathcal{E}_{i}$.

Both Deontic Supervaluationism and Deontic Subvaluationism allow for non-trivial deontic distinctions in certain cases in which $\mathbf{O}^{U}$ is non-empty. ${ }^{18}$ Moreover, these seem to me to be the only principled ways of deriving such a distinction that satisfy the above constraints.

There is, however, good reason to reject Deontic Supervaluationism. For Deontic Supervaluationism together with Maximization and Global Dominance Permissivism entails that if $\mathbf{O}^{U}$ is singleton and, for each $O \in \mathbf{O}^{D}$, there is some $O^{\prime} \in \mathbf{O}^{D}$, such that $E C U_{i}\left(O^{\prime}\right)>E C U_{i}(O)$, then one is rationally required to realise the unique $O \in O^{U} \cdot{ }^{19}$ This consequence is, I take it, a reductio of the conjunction of these three views. For surely an agent should not be rationally required to realize the unique option about which they lack a view regarding its expected causal utility. Given the plausibility of Maximization and Global Dominance Permissivism, then, this provides us with good reason to reject Deontic Supervaluationism.

I don't think, though, that we have any such clear reason to reject Deontic Subvaluationism. There would seem, then, to be a defensible view on which non-trivial deontic distinctions may sometimes be drawn amongst an agent's options even if the agent lacks well-defined expectation values for some of their available options. Importantly, though, given Global Dominance Permissivism, such non-trivial distinctions can only be drawn when

\footnotetext{
${ }^{18}$ To see this in the case of Deontic Subvaluationism, suppose that $O^{U}$ is finite and that there is some subset of $O^{D}$ that is maximal given $E C U_{i}(\cdot)$. Then, given Deontic Subvaluationism the only permissible options will be the members of $O^{U}$ and the maximal members of $O^{D}$. Below, I'll consider a case in which Deontic Supervaluationism provides for non-trivial deontic distinctions given non-empty $\mathbf{O}^{U}$.

${ }^{19}$ To see this, let $O^{*}$ be the unique member of $\mathbf{O}^{U}$. Given that $O^{*}$ is the unique member of $\mathbf{O}^{U}$ and that, for each $O \in \mathbf{O}^{D}$, there is some $O^{\prime} \in \mathbf{O}^{D}$, such that $E C U_{i}\left(O^{\prime}\right)>E C U_{i}(O)$, it follows that (i) there exists some $E C U_{i}^{*}(\cdot) \in \mathcal{E}_{i}$ such that $E C U_{i}^{*}\left(O^{*}\right)>E C U_{i}^{*}(O)$, for each other $O \in \mathbf{O}_{i}^{t^{\prime}}$, and (ii) every $E C U_{i}^{*}(\cdot) \in \mathcal{E}_{i}$ that does not satisfy the preceding condition is such that, for each $O \in \mathbf{O}_{i}^{t^{\prime}}$, there is some $O^{\prime} \in \mathbf{O}_{i}^{t^{\prime}}$, such that $\operatorname{ECU}_{i}^{*}\left(O^{\prime}\right)>E C U_{i}^{*}(O)$. Given Deontic Supervaluationism and Maximization, it follows from (i) that every $O \in \mathbf{O}^{D}$ is impermissible. And given Deontic Supervaluationism, Maximization and Global Dominance Permissivism, it follows from (i) and (ii) that $O^{*}$ is permissible. And so, since $O^{*}$ is the unique permissible option amongst $\mathbf{O}_{i}^{t^{\prime}}$, given Deontic Supervaluationism, Maximization and Global Dominance Permissivism, it follows, given these principles, that it is rationally obligatory that $i$ realize $O^{*}$.
} 
$\mathrm{O}^{U}$ is finite. For Global Dominance Permissivism and Deontic Subvaluationism entail that if $E C U_{i}(\cdot)$ is not well-defined for an infinite $\mathbf{O}^{U} \subseteq \mathbf{O}_{i}^{t^{\prime}}$, then, for each $O \in \mathbf{O}_{i}^{t^{\prime}}$, it is rationally permissible for $i$ to realize $O .^{20}$

Since Deontic Subvaluationism is the only reasonable way of drawing non-trivial deontic distinctions in certain cases in which $\mathbf{O}^{U}$ is non-empty that satisfies certain plausible constraints, and since we have good reason to accept Global Dominance Permissivism, we have, then, good reason to think that if there is a way of drawing non-trivial deontic distinctions in certain cases in which $\mathrm{O}^{U}$ in non-empty, then if $E C U_{i}(\cdot)$ is not well-defined for an infinite $\mathbf{O}^{U} \subseteq \mathbf{O}_{i}^{t^{\prime}}$, then, for each $O \in \mathbf{O}_{i}^{t^{\prime}}$, it is rationally permissible for $i$ to realize $O$.

A consequence of this conditional is that if there is a way of drawing non-trivial deontic distinctions in certain cases in which $\mathrm{O}^{U}$ in non-empty, such distinctions can only be drawn when $\mathbf{O}^{U}$ is finite. And so it follows that any case in which $\mathbf{O}^{U}$ is infinite will be such that either each option is permissible, or each option is impermissible, or each option is not apt for rational evaluation. Thus, we have No Distinctions.

Now, if one thinks that non-trivial deontic distinctions can be drawn at least in certain cases in which $\mathrm{O}^{U}$ is non-empty, then the preceding also provides us with an argument that if $E C U_{i}(\cdot)$ is not well-defined for an infinite $\mathbf{O}^{U} \subseteq \mathbf{O}_{i}^{t^{\prime}}$, then, for each $O \in \mathbf{O}_{i}^{t^{\prime}}$, it is rationally permissible for $i$ to realize $O$. But one might reject the claim that non-trivial deontic distinctions can ever be drawn in cases in which $\mathbf{O}^{U}$ is non-empty, and so reject this argument for the claim that when $\mathbf{O}^{U}$ is infinite each option available to the agent is rationally permissible. For example, one may maintain that if $\mathbf{O}^{U}$ is non-empty, then each option available to the agent is not apt for rational assessment. Or one may maintain that if $\mathbf{O}^{U}$ is non-empty, then each option available to the agent is rationally impermissible. We need not, though, consider how plausible any of these particular claims are. For No Distinctions itself will suffice for our purposes in what follows.

\section{Credal Consequentialism}

According to credal consequentialism, the deontic status of an agent's credal options is determined by the degree to which the agent takes such options to be conducive to the epistemic good. In this section, we'll sharpen up this intuitive idea and look more closely at the picture of credal rationality that it provides.

In $\S 2.1$, I'll outline, by appeal to the machinery of causal decision theory, a precise version of credal consequentialism. In $\S 2.2$, I'll show that, given plausible auxiliary assumptions, this theory has the undesirable consequence that in a vast range of cases no deontic distinctions can be drawn amongst an agent's credal options. In $\S 3$, I'll then consider two ways in which one might try to avoid this result.

${ }^{20}$ To see this, assume that $\mathbf{O}^{U}$ is infinite. Then it follows that there is some $E C U_{i}^{*}(\cdot) \in \mathcal{E}_{i}$, such that, for each $O \in \mathbf{O}_{i}^{t^{\prime}}$, there is some $O^{\prime} \in \mathbf{O}_{i}^{t^{\prime}}$, such that $E C U_{i}^{*}\left(O^{\prime}\right)>E C U_{i}^{*}(O)$. And so, given Global Dominance Permissivism, each $O \in \mathbf{O}_{i}^{t^{\prime}}$ is permissible relative to $E C U_{i}^{*}(\cdot)$. And so, given Deontic Subvaluationism, it follows that each $O \in \mathbf{O}_{i}^{t^{\prime}}$ is permissible. 


\subsection{Credal Consequentialism Precisified}

We'll assume that there is an objective notion of the epistemic good for credal states that may be represented by a ternary function $e u(\cdot, \cdot, \cdot): I \times T \times W \rightarrow \mathbb{R}$. Here $I$ is the set of possible individuals, $T$ is the set of possible times, and $W$ is the set of epistemically possible worlds. Given an individual $i$, a time $t$, and a world $w, e u(i, t, w)$, then, tells us the epistemic utility of $i$ 's credal state at $w$ and $t$. As a short-hand, we'll let $e u_{i}^{t}(\cdot)=e u(i, t, \cdot) .{ }^{21}$

Let $\mathbf{C}_{i}^{t^{\prime}}$ be the partition of $W$ consisting of $i$ 's credal options at $t^{\prime}$. We'll assume that the agent's conceptual resources at $t^{\prime}$ are an exogenous factor, outside of their control, at least in the sense that is relevant for credal decision problems. Thus, we'll assume that there is a fixed algebra $\mathbf{A}_{i}^{t^{\prime}}$ such that the agent's credal options at $t^{\prime}$ concern possible credal states defined over $\mathbf{A}_{i}^{t^{\prime}}$, and that this algebra is the same algebra over which the agent's stable credences leading up to $t^{\prime}$ are defined, so that for some $t^{\prime \prime}<t^{\prime}$, for each $t \in\left(t^{\prime \prime}, t^{\prime}\right), \mathbf{A}_{i}^{t}=\mathbf{A}_{i}^{t^{\prime}}$.

Even given these constraints, it turns out to be a somewhat delicate matter how we should think of the elements of $\mathbf{C}_{i}^{t^{\prime}}$. A natural thought is that the elements of $\mathbf{C}_{i}^{t^{\prime}}$ will be propositions uniquely characterizing $i$ 's credal distribution, at $t^{\prime}$, over $\mathbf{A}_{i}^{t^{\prime}}$. In this section, we'll operate under this assumption. In the next section, though, we'll consider the possibility that the members of $\mathbf{C}_{i}^{t^{\prime}}$ may consist of coarse-grainings of this space of propositions, so that each $C \in \mathbf{C}_{i}^{t^{\prime}}$ may be thought of as a union of propositions characterizing $i$ 's credal distribution over $\mathbf{A}_{i}^{t^{\prime}}$ at $t^{\prime} .{ }^{22}$

\footnotetext{
${ }^{21} \mathrm{~A}$ few points. First, note that the notion of utility appealed to here is not tied to an agent's subjective values. Nonetheless, we will assume that it can be known a priori that the epistemic good may be represented by $e u(\cdot, \cdot, \cdot)$. For, otherwise, it is hard to see how $e u(\cdot, \cdot, \cdot)$ could have the deontic bearing that it has according to the version of credal consequentialism under consideration.

Second, one might worry about those cases in which $i$ does not exist at $w$ and $t$. There are two ways of dealing with such cases. Either we could take $e u(\cdot, \cdot, \cdot)$ to be a partial function that is only defined when $i$ exists at $w$ and $t$. Or we could take the epistemic utility of having no credal state to be some particular value. Assuming that there is a minimal epistemic utility that one could have were one to have a credal state, a natural option, here, would be to take the epistemic utility of failing to have any credal state whatsoever to be a value less than this minimal value. For our purposes, it won't really matter which of these options we choose. To simplify the exposition, though, I'll ignore this complication in what follows.

Third, while we will assume that the notion of the epistemic good that is relevant to credal consequentialism is objective and independent of any agent's values, it should be clear that almost all of the main points that follow could be made equally well given a more subjective version of credal consequentialism. Typically, though, insofar as pursuit of the epistemic good is thought to be relevant to epistemology, it is an objective notion the good that is taken to be so relevant.

${ }^{22}$ A worry: Given this way of characterizing $\mathbf{C}_{i}^{t^{\prime}}$, it wouldn't seem that this set will be a partition of $W$. For, in addition to all the worlds in which $i$ has some credal state defined over $\mathbf{A}_{i}^{t^{\prime}}$ at $t^{\prime}$, there are all sorts of other worlds in which $i$ does not have a credal state at $t^{\prime}$ defined over $\mathbf{A}_{i}^{t^{\prime}}$.

To get around this worry, we weasel. Let $\mathbf{C}^{*}$ be the set of propositions describing various possible credal states that $i$ may have at $t^{\prime}$ over $\mathbf{A}_{i}^{t^{\prime}}$, or a set of disjoint unions of such propositions the union of which is equivalent to the union of all of the propositions in the former set. Now pick an arbitrary $C \in \mathbf{C}^{*}$. Then $\mathbf{C}^{*}-\{C\}$ is the set of propositions in $\mathbf{C}^{*}$ with $C$ removed. And so $\left(W-\cup\left(\mathbf{C}^{*}-\{C\}\right)\right)$ is the proposition that is true just in case it's not the case that any of the propositions in $\mathbf{C}^{*}-\{C\}$ are true. Now let $\mathbf{C}_{i}^{t^{\prime}}=\left(\mathbf{C}^{*}-\{C\}\right) \cup\left(W-\cup\left(\mathbf{C}^{*}-\{C\}\right)\right)$. $\mathbf{C}_{i}^{t^{\prime}}$, then, is the result of replacing $C$ in $\mathbf{C}^{*}$ with the proposition that none of the other propositions in $\mathbf{C}^{*}$ obtain. $\mathbf{C}_{i}^{t^{\prime}}$, so characterized, will be guaranteed to be a partition
} 
Let $P$ be a set such that, for some $x \in \mathbb{R}, P=\left\{w \in \mathbf{W}: e u_{i}^{t^{\prime}}(w)=x\right\}$. We'll call such a set an epistemic value proposition (for $i$ at $t^{\prime}$ ), and we'll denote such a proposition by $\left[E V_{i}^{t^{\prime}}=x\right]$, and the class of such propositions by $\mathbf{E V}_{i}^{t^{\prime}}$. Given some $C \in \mathbf{C}_{i}^{t^{\prime}}$ and some $\left[E V_{i}^{t^{\prime}}=x\right] \in \mathbf{E V}_{i}^{t^{\prime}}, C \square \rightarrow\left[E V_{i}^{t^{\prime}}=x\right]$, then, is the set of worlds $w$ such that, at $w$, were $i$ to realize $C$ at $t^{\prime}$, then the result would be a situation of epistemic utility $x$.

We'll say that a non-empty intersection of a set that contains, for each $C \in \mathbf{C} \subseteq \mathbf{C}_{i}^{t^{\prime}}$, a unique proposition of the form $C \square \leftrightarrow\left[E V_{i}^{t^{\prime}}=x\right]$, and no other propositions, is an epistemicvalue dependence hypothesis for $\mathbf{C}$. We'll denote the set of epistemic-value dependence hypotheses for $\mathbf{C} \subseteq \mathbf{C}_{i}^{t^{\prime}}, \mathbf{E D}(\mathbf{C})$.

Let us say, for each $C \in \mathbf{C}_{i}^{t^{\prime}}$ and each $P \subseteq \mathbf{A}_{i}^{t}$, that $E U(C \cap P)=x$ if $\left(C \square \mapsto\left[E V_{i}^{t^{\prime}}=\right.\right.$ $x]) \cap P=P$, and otherwise $E U(C \cap P)$ is undefined. Given this characterization of $E U(\cdot)$, we can define the following quantity:

$$
\text { Def. } E G_{i}^{t}(C)={ }_{d f} \sum_{A \in \mathbf{A t}_{i}^{t}} C r_{i}^{t}(A) E U(C \cap A)
$$

For the reasons canvassed in $\$ 1$, we won't, in general, want to identify an agent's expectation value for the casual epistemic utility of some option $C$ with $E G_{i}^{t}(C)$. Nonetheless, this quantity will play an important role in what follows, and so we single it out.

We'll instead take the expected epistemic causal utility, for $i$ at $t$, of an option $C \in \mathbf{C}_{i}^{t^{\prime}}$ to be characterized as follows:

$$
\text { EECU: } E E C U_{i}^{t}(C)=\sum_{A \in \mathbf{Z}_{C r_{i}^{t}}} C r_{i}^{t}(A) E U(C \cap A)
$$

And, given this characterization of expected epistemic utility, we can then define the following, perhaps partial, function from $\mathbf{C}_{i}^{t^{\prime}}$ to $\mathbb{R}$ :

Def. $E E C U_{i}(C)=x$ just in case, for all $t \in\left(t^{\prime \prime}, t^{\prime}\right), E E C U_{i}^{t}(C)=x$

Again, given our assumption that, in a given credal decision problem, there will be some $t^{\prime \prime}<t^{\prime}$, such that, for each $t_{1}, t_{2} \in\left(t^{\prime \prime}, t^{\prime}\right), C r_{i}^{t_{1}}(\cdot)=C r_{i}^{t_{2}}(\cdot), E E C U_{i}(C)$ will be well-defined as long at $E E C U_{i}^{t}(C)$ is well-defined for some $t \in\left(t^{\prime \prime}, t^{\prime}\right)$.

The core idea behind credal consequentialism, I take it, is that one should try to realize a credal option that will bring about the best epistemic consequences. Or, rather, if there are credal options that maximize an agent's expected epistemic utility, then the agent ought to realize one of those options. As with the core idea behind causal decision theory, there are a few ways in which this idea may be precisified. In what follows, we'll work with the following natural precisification:

of $W$. And, given that the agent is certain that, at $t^{\prime}$, they will have credences defined over $\mathbf{A}_{i}^{t^{\prime}}, C$ and $\left(W-\cup\left(\mathbf{C}^{*}-\{C\}\right)\right)$ will play the same role in the agent's credal state.

This being said, I'll ignore this complication in what follows and speak of the members of $\mathbf{C}_{i}^{t^{\prime}}$ as if they characterize (perhaps disjunctively) the agent's credences at $t^{\prime}$ over $\mathbf{A}_{i}^{t^{\prime}}$. 
Credal Maximization: Let $M$ be the set of $C \in \mathbf{C}_{i}^{t^{\prime}}$ such that, for each $C^{\prime} \in \mathbf{C}_{i}^{t^{\prime}}$, $E E C U_{i}(C) \geq E E C U_{i}\left(C^{\prime}\right)$. If $M$ is non-empty, then, for each $C \in M$, it is rationally permissible for $i$ to realize $C$, and it is rationally required for $i$ to realize some $C \in M^{23}$

Credal Maximization, of course, leaves open the deontic status of an agent's credal options in two important classes of cases. First, it leaves open the deontic status of an agent's credal options when, for each credal option, there is another credal option with greater expected causal epistemic utility. Second, it leaves open the deontic status of an agent's credal options when there is no well-defined causal expectation value for at least some credal options.

The arguments presented in $\S 1$, though, tell us what the proponent of credal consequentialism ought to say about the first class of cases and at least a subclass of the second class of cases. In particular, the arguments presented in $\S 1$ tell us that, in addition to Credal Maximization, the proponent of credal consequentialism should endorse:

Global Credal Dominance Permissivism: If, for each credal option $C$, there is some option $C^{\prime}$, such that $E E C U_{i}\left(C^{\prime}\right)>E E C U_{i}(C)$, then, for each $C \in \mathbf{C}_{i}^{t^{\prime}}$, it is rationally permissible for $i$ to realize $C$.

No Credal Distinctions: If $E E C U_{i}(\cdot)$ is not well-defined for an infinite $\mathbf{C}^{U} \subseteq \mathbf{C}_{i}^{t^{\prime}}$, then, either, for each $C \in \mathbf{C}_{i}^{t^{\prime}}$, it is rationally permissible for $i$ to realize $C$, or, for each $C \in \mathbf{C}_{i}^{t^{\prime}}$, it is rationally impermissible for $i$ to realize $C$, or, for each $C \in \mathbf{C}_{i}^{t^{\prime}}$, it is neither rationally permissible nor rationally impermissible for $i$ to realize $C$.

For, in $\S 1$, we argued that, given Maximization, one should endorse Global Dominance Permissivism. And this argument may be repurposed, mutatis mutandis, to show that, given Credal Maximization, one should endorse Global Credal Dominance Permissivism. And, in $\S 1$,

${ }^{23}$ The natural generalization of Credal Maximization is:

Diachronic Credal Maximization: Let $M$ be the set of $C \in \mathbf{C}_{i}^{t^{\prime}}$ such that, for some $t^{\prime \prime}<t^{\prime}$, $E E C U_{i}^{t}(C) \geq E C U_{i}^{t}\left(C^{\prime}\right)$, for every $C^{\prime} \in \mathbf{C}_{i}^{t^{\prime}}$ and every $t \in\left(t^{\prime \prime}, t^{\prime}\right)$. If $M$ is non-empty, then, for each $C \in M$, it is rationally permissible for $i$ to realize $C$, and it is rationally required for $i$ to realize some $C \in M$.

An alternative way of precisifying the core idea behind credal consequentialism is:

Synchronic Credal Maximization: Let $M$ be the set of $C \in \mathbf{C}_{i}^{t^{\prime}}$ such that, for each $C^{\prime} \in$ $\mathbf{C}_{i}^{t^{\prime}}, E E C U_{i}^{t^{\prime}}(C) \geq E E C U_{i}^{t^{\prime}}\left(C^{\prime}\right)$. If $M$ is non-empty, then, for each $C \in M$, it is rationally permissible for $i$ to realize $C$, and it is rationally required for $i$ to realize some $C \in M$.

Earlier, I noted some reasons to prefer Maximization to its synchronic alternative Synchronic Credal Maximization. There are also, I think, corresponding reasons to prefer Credal Maximization to Synchronic Credal Maximization. However, deciding between these different ways of precisifying the guiding thought behind credal consequentialism need not detain us here. For all of the main points that I'll make have natural analogues given the natural alternative version of credal consequentialism that endorses Synchronic Credal Maximization instead of Credal Maximization. 
we argued that, given Global Dominance Permissivism, one should endorse No Distinctions. And this argument may be repurposed, mutatis mutandis, to show that, given Global Credal Dominance Permissivism, one should endorse No Credal Distinctions.

\subsection{A Problem for Credal Consequentialism}

I'll now argue that, in a large class of cases, credal consequentialism entails that no nontrivial deontic distinctions can be drawn amongst an agent's credal options. The problem, in essence, is that, given a typical algebra $\mathbf{A}_{i}^{t^{\prime}}$, there are simply more -indeed infinitely many more - dependence hypotheses for the different credal states over $\mathbf{A}_{i}^{t^{\prime}}$ that an agent $i$ might adopt at $t^{\prime}$ than there are atoms of $\mathbf{A}_{i}^{t^{\prime}}$. Given that $i$ has credences defined over $\mathbf{A}_{i}^{t^{\prime}}$, leading up to $t^{\prime}$, the agent, then, simply can't form an appropriate view about the expectation values for the various credal states that they may adopt at $t^{\prime}$ over $\mathbf{A}_{i}^{t^{\prime}}$ - or at least they can't do so without ruling out infinitely many a priori possible epistemic dependence hypotheses. And given this consequence it follows from No Credal Distinctions that, in all but some very unusual cases, unless an agent rules out an infinite number of a priori possible epistemic dependence hypotheses, credal consequentialism will entail that no non-trivial deontic distinctions can be drawn amongst an agent's credal options.

The first point to note is that, in any case in which an agent has credences defined over an algebra of propositions that don't concern their own credal states, it follows, given No Credal Distinctions, that no non-trivial deontic distinctions can be drawn amongst an agent's credal options.

Let me briefly sketch why this is so by appeal to a toy case.

Rain: Let $R=\left\{w\right.$ : It is raining in $w$ at $\left.t^{\prime}\right\}$ and $\mathbf{A}^{R}=\{R, W-R, \emptyset, W\} . \mathbf{A}^{R}$, then, is the smallest algebra containing $R$. And let $\mathbf{C}^{R}$ be the set of propositions that uniquely characterize the possible credal distributions that $i$ may have at $t^{\prime}$ over $\mathbf{A}^{R}$.

Claim: Given No Credal Distinctions, no non-trivial deontic distinctions can be drawn amongst the agent's different credal options in Rain.

Justification: Each $C \in \mathbf{C}^{R}$ is compatible with both the truth of $R$ and the falsity of $R$. Moreover, given any plausible account of epistemic value, we should expect that the class of $C \in \mathbf{C}^{R}$ for which the epistemic utility of $C$ given the truth of $R$ does not differ from the epistemic utility of $C$ given the falsity of $R$ will be quite small. ${ }^{24}$ In particular, given that there are an infinite number of $C \in \mathbf{C}^{R}$, we should expect that this set will, at the very least, be non-cofinite. Thus, the class of $C \in \mathbf{C}^{R}$ for which the epistemic utility of $C$ given the truth of $R$ does differ from the epistemic utility of $C$ given the falsity of $R$ is infinite. Furthermore, we should expect that, for each $C \in \mathbf{C}^{R}$, in addition to the mundane

${ }^{24}$ The sorts of symmetries that give rise to cases in which the epistemic utility of a credal state may be invariant given such differences in truth-value are further discussed below. 
worlds in $R$, in which $C \square \rightarrow R$ holds, there will also be some rather odd worlds in which $C \square \rightarrow(W-R)$ holds. Similarly, we should expect that, for each $C \in \mathbf{C}^{R}$, in addition to the mundane worlds in $W-R$, in which $C \square \rightarrow(W-R)$ holds, there will also be some rather odd worlds in which $C \square \rightarrow R$ holds. It follows, then, that for an infinite number of $C \in \mathbf{C}^{R}$ both $E U(C \cap R)$ and $E U(C \cap(W-R))$ will be undefined. And so we have that $E E C U_{i}(\cdot)$ is not well-defined for an infinite $\mathbf{C}^{U} \subseteq \mathbf{C}^{R}$. Thus, given No Credal Distinctions, it follows that either, for each $C \in \mathbf{C}^{R}$, it is rationally permissible for $i$ to realize $C$, or, for each $C \in \mathbf{C}^{R}$, it is rationally impermissible for $i$ to realize $C$, or, for each $C \in \mathbf{C}^{R}$, it is neither rationally permissible nor rationally impermissible for $i$ to realize $C$.

While I think that this consequence is quite undesirable, there are a few responses available to the proponent of credal consequentialism. For example, they may maintain that epistemic rationality requires an agent to form an appropriate view about the expected epistemic utility of their own credences. In this way, they may try to justify the claim that, in this sort of case, all of an agent's credal options are rationally impermissible. Or they may maintain that, in order to assess an agent's credal state as being rationally permissible or impermissible, the agent must form an appropriate view about the expected epistemic utility of their own credences. In this way, they may try to justify the claim that, in this sort of case, none of the agent's credal options can be assessed as either being rationally permissible or rationally impermissible.

For this sort of move to be at all persuasive, though, there must be a sufficiently large class of cases in which non-trivial deontic distinctions may be drawn, given credal consequentialism. In this section, I'll argue, however, that this condition isn't satisfied. To this end, I'll argue that, given No Credal Distinctions, in all but some unusual cases, we have:

Opinionation: No non-trivial deontic distinctions can be drawn amongst an agent's credal options, unless $i$ is quasi-opinionated about $\mathbf{E D}(\{C\})$, for an infinite number of $C \in \mathbf{C}_{i}^{t^{\prime}}$, in the stable interval leading up to $t^{\prime}$.

To argue for this claim, I'll first argue that, in all but some unusual cases, we have:

Undefinedness: If $\mathbf{C}_{i}^{t^{\prime}}$ is the set of propositions characterizing $i$ 's possible credal distributions, at $t^{\prime}$, over $\mathbf{A}_{i}^{t^{\prime}}$, then there will be some infinite $\mathbf{C} \subseteq \mathbf{C}_{i}^{t^{\prime}}$ such that, for each $C \in \mathbf{C}$, there is some $A \in \mathbf{A t}_{i}^{t^{\prime}}$, for which $E U(C \cap A)$ is undefined.

Given Undefinedness, it follows that, for each $t$ in the stable interval leading up to $t^{\prime}$, $E G_{i}^{t}(C)$ will be undefined for each $C \in \mathbf{C}$. And from this it follows that, unless, in the stable interval leading up to $t^{\prime}, i$ is quasi-opinionated about $\operatorname{ED}(\{C\})$, for an infinite number of $C \in \mathbf{C}_{i}^{t^{\prime}}, E E C U_{i}(\cdot)$ will not be well-defined for an infinite $\mathbf{C}^{U} \subseteq \mathbf{C}_{i}^{t^{\prime}}$. And so, given that Undefinedness holds in all but some unusual cases, it follows, given No Credal Distinctions, that Opinionation holds in all but some unusual cases.

In support of the claim that Undefinedness holds in all but some unusual cases, I'll first argue that, in all but some unusual cases, the following two claims will both hold: 
Bifurcation: Let $\mathbf{C}_{\geq 2}\left(\mathbf{C}_{i}^{t^{\prime}}\right) \subseteq \mathbf{C}_{i}^{t^{\prime}}$ be the set of $C \in \mathbf{C}_{i}^{t^{\prime}}$ such that $|\mathbf{E D}(\{C\})| \geq$ 2 . If $\mathbf{C}_{i}^{t^{\prime}}$ is the set of propositions uniquely characterizing $i$ 's possible credal distributions over $\mathbf{A}_{i}^{t^{\prime}}$, then $\left|\mathbf{C}_{\geq 2}\left(\mathbf{C}_{i}^{t^{\prime}}\right)\right|$ will be infinite and will be such that $\left|\mathbf{C}_{\geq 2}\left(\mathbf{C}_{i}^{t^{\prime}}\right)\right| \geq\left|\mathbf{A}_{i}^{t^{\prime}}\right|$

Exponentiation: For each $\mathbf{C} \subseteq \mathbf{C}_{\geq 2}\left(\mathbf{C}_{i}^{t^{\prime}}\right),|\mathbf{E D}(\mathbf{C})| \geq 2^{|\mathbf{C}|}$.

As we'll show, given that both of these claims hold in all but some unusual cases, it follows that Undefinedness will hold in all but some unusual cases.

Let's begin with Bifurcation. To see why it is plausible that this claim holds in typical cases, it will be helpful to first consider an unusual case in which the set of credal options, $\mathbf{C}_{T(*)}$, is such that $\left|\mathbf{C}_{\geq 2}\left(\mathbf{C}_{T(*)}\right)\right|=0 .{ }^{25}$

Anti-Expert: Consider the following interpreted sentence: $(*)$ It's not the case that $i$ 's credence at $t^{\prime}$ that $(*)$ is true is greater than or equal to 0.5. We can represent this as: $(*) \neg C r_{i}^{t^{\prime}} T(*) \geq 0.5$. Let $\mathbf{A}_{T(*)}=\{T(*), W-T(*), \emptyset, W\}$. $\mathbf{A}_{T(*)}$, then, is the smallest algebra containing $T(*)$. Let $\mathbf{C}_{T(*)}$ be the set of propositions that uniquely characterize the possible credal distributions that $i$ may have at $t^{\prime}$ over $\mathbf{A}_{T(*)}$.

Now the following principle concerning epistemic utility seems to me to be quite plausible:

Truth-Value Supervenience: For every $w, w^{\prime} \in W, t, t^{\prime} \in T$, and $i, i^{\prime} \in I$, if $\mathbf{A}_{i}^{t}=\mathbf{A}_{i^{\prime}}^{t^{\prime}}, \operatorname{Cr}_{i}^{t}(\cdot)=C r_{i^{\prime}}^{t^{\prime}}(\cdot)$ and the truth-value distribution at $w$ over $\mathbf{A}_{i}^{t}$ is the same as the truth-value distribution at $w^{\prime}$ over $\mathbf{A}_{i^{\prime}}^{t^{\prime}}$, then $e u(i, t, w)=e u\left(i^{\prime}, t^{\prime}, w^{\prime}\right)$.

Claim: Given Truth-Value Supervenience, it follows that, in Anti-Expert, for each $C \in \mathbf{C}_{T(*)}$, there is a particular epistemic utility value such that necessarily that value would result were $C$ to be realized. That is, given Truth-Value Supervenience, $\mathbf{C}_{\geq 2}\left(\mathbf{C}_{T(*)}\right)=\emptyset$.

Justification: Given Truth-Value Supervenience, it follows that, for each $C \in$ $\mathbf{C}_{T(*)}$, there exists some $x \in \mathbb{R}$, such that $C \square \leftrightarrow\left[E V_{i}^{t^{\prime}}=x\right]$ holds at $w$, for each $w \in \mathbf{W}$. For note that as an instance of the T-schema, we have: $T(*) \leftrightarrow$ $\neg C r_{i}^{t^{\prime}} T(*) \geq 0.5$. Since this proposition is both necessary and a priori knowable, it will obtain at every point in $W .{ }^{26}$ And, given that $T(*) \leftrightarrow \neg C r_{i}^{t^{\prime}} T(*) \geq 0.5$ obtains at each $w \in W$, it follows that, for each $C r(\cdot): \mathbf{A}_{T(*)} \rightarrow \mathbb{R}$, each $w \in W$ will agree about what the truth-value distribution would be over $\mathbf{A}_{T(*)}$ were $i$ at $t^{\prime}$ to have credal state $C r(\cdot)$. And so, given Truth-Value Supervenience, it follows that each $w \in \mathbf{W}$ will agree, for each $C r(\cdot): \mathbf{A}_{T(*)} \rightarrow \mathbb{R}$, about how much

${ }^{25}$ This sort of case is considered in detail in Caie (2013) and Caie (2014). For further discussion of such cases see Campbell-Moore (2015). And for a treatment of such cases in a non-classical setting see Caie (2012).

${ }^{26}$ See Caie (2013) for a defense of the claim that this proposition is both necessary and a priori. 
epistemic utility would result were $i$ at $t^{\prime}$ to have credal state $\operatorname{Cr}(\cdot)$. And so, given this plausible principle concerning epistemic utility, it follows that there is a single epistemic-value dependence hypothesis for $\mathbf{C}_{T(*)}$, viz., $W$. And so, we have $\mathbf{C}_{\geq 2}\left(\mathbf{C}_{T(*)}\right)=\emptyset$.

It's worth stressing, though, that the pair of $\mathbf{C}_{T(*)}$ and $\mathbf{A}_{T(*)}$ have a very unusual property. For each member of $\mathbf{C}_{T(*)}$ entails a particular truth-value distribution over $\mathbf{A}_{T(*)}$. Given a typical algebra, $\mathbf{A}$, though, there will be no agent $i$ and time $t^{\prime}$ such that each proposition that describes $i$ 's credal distribution over $\mathbf{A}$ at $t^{\prime}$ entails a particular truth-value distribution over A. Indeed, for a typical algebra, $\mathbf{A}$, any agent $i$ and time $t^{\prime}$ will be such that no proposition that describes $i$ 's credal distribution over $\mathbf{A}$ at $t^{\prime}$ will entail a particular truthvalue distribution over $\mathbf{A}$.

Thus, suppose that to $\mathbf{A}_{T(*)}$ we add the proposition $R=\left\{w\right.$ : It is raining in $w$ at $\left.t^{\prime}\right\}$ and close under negation and disjunction. Let $\mathbf{A}_{T(*)}^{R}$ denote the resulting algebra, and let $\mathbf{C}_{T(*)}^{R}$ be the set of propositions that characterize the possible credal distributions that $i$ may have at $t^{\prime}$ over $\mathbf{A}_{T(*)}^{R}$. Then, unlike with $\mathbf{C}_{T(*)}$ and $\mathbf{A}_{T(*)}$, no member of $\mathbf{C}_{T(*)}^{R}$ entails a particular truth-value distribution over $\mathbf{A}_{T(*)}^{R}$. For while each $C \in \mathbf{C}_{T(*)}^{R}$ will entail a truthvalue distribution over the sub-algebra $\mathbf{A}_{T(*)}$, each $C \in \mathbf{C}_{T(*)}^{R}$ is compatible with both the truth and the falsity of $R$.

Now we can't, I think, conclude from this fact that every $C \in \mathbf{C}_{T(*)}^{R}$ may result in more than one possible epistemic utility. For, while each $C \in \mathbf{C}_{T(*)}^{R}$ is compatible with at least two distinct truth-value distributions over $\mathbf{A}_{T(*)}^{R}$, given plausible principles concerning epistemic utility, for some $C \in \mathbf{C}_{T(*)}^{R}$ there will be symmetries that ensure that the same epistemic utility will result given $C$ and any of the possible truth-value distributions over $\mathbf{A}_{T(*)}^{R}$ compatible with $C$. For example, suppose that $C$ describes $i$ at $t^{\prime}$ as having a probabilistically coherent credal state $C r_{i}^{t^{\prime}}(\cdot)$ such that $C r_{i}^{t^{\prime}}(T(*))>0.5$ and such that $C r_{i}^{t^{\prime}}(R)=C r_{i}^{t^{\prime}}(W-R)=0.5$. In this case, there will be two possible truth-value distributions over $\mathbf{A}_{T(*)}^{R}$ compatible with $C$, viz., the truth-value distribution determined by the falsity of $T(*)$ and the truth of $R$, and the truth-value distribution determined by the falsity of $T(*)$ and the falsity of $R$. However, given that $\operatorname{Cr}_{i}^{t^{\prime}}(R)=C r_{i}^{t^{\prime}}(W-R)=0.5$ and that $\operatorname{Cr}_{i}^{t^{\prime}}(\cdot)$ is probabilistically coherent, it seems quite reasonable to suppose that were $C$ to obtain, the same epistemic utility would result, regardless of which of these two truth-value distributions obtained. ${ }^{27}$

I think, though, that this sort of symmetry will be the exception rather than the rule, given any plausible account of epistemic utility. Thus, for example, if $C^{\prime}$ describes $i$ at $t^{\prime}$ as having a probabilistically coherent credal state $\operatorname{Cr}_{i}^{t^{\prime}}(\cdot)$ such that $\operatorname{Cr}_{i}^{t^{\prime}}(T(*))>0.5$ and

\footnotetext{
${ }^{27}$ This, for example, will hold if, in cases in which an agent $i$ at $w$ and $t$ has a credal state defined over a finite algebra, we take $e u(t, i, w)$ to be given by the negative Brier score. Let $C r(\cdot)$ give the agent's credences at $w$ and $t$ over an $n$-membered algebra $\mathbf{A}$. And let $w(\cdot): \mathbf{A} \rightarrow\{0,1\}$ be such that $w(P)=1$ just in case $P$ is true at $w$. Then, the negative Brier score for $i$ 's credences at $w$ and $t$ is given by:

$$
1-\left[(1 / n) \sum_{P \in \mathbf{A}}(C r(P)-w(P))^{2}\right]
$$
}


such that $C r_{i}^{t^{\prime}}(R)>0.5$, then, while the same two possible truth-value distributions that were compatible with $C$ will be compatible with $C^{\prime}$, it seems that greater epistemic utility would result were the conjunction of $C^{\prime}$ and the truth-value distribution determined by the falsity of $T(*)$ and the truth of $R$ to obtain, instead of the conjunction of $C^{\prime}$ and the truthvalue distribution determined by the falsity of $T(*)$ and the falsity of $R .{ }^{28}$ Given, then, a reasonable account of epistemic utility, we will have that the class of $C \in \mathbf{C}_{T(*)}^{R}$ for which there are special symmetries that ensure that there is only one epistemic utility value that could result were $C$ to be realized will be relatively small. And, given that $\mathbf{C}_{T(*)}^{R}$ is infinite, this set will, at the very least, be non-cofinite. Thus, we have that the class of $C \in \mathbf{C}_{T(*)}^{R}$ for which more than one possible epistemic utility value may result were $C$ to obtain will be infinite.

The preceding considerations, moreover, readily generalize. In all but some very strange cases, each $C \in \mathbf{C}_{i}^{t^{\prime}}$ will be compatible with at least two possible truth-value distributions over $\mathbf{A}_{i}^{t^{\prime}}$. This, for example, will be assured if, like $\mathbf{A}_{T(*)}^{R}, \mathbf{A}_{i}^{t^{\prime}}$ contains at least one proposition that is logically independent of any credal state over $\mathbf{A}_{i}^{t^{\prime}}$ that $i$ might have at $t^{\prime}$. And, in such cases, with the exception of a relatively small, non-cofinite, number of $C \in \mathbf{C}_{i}^{t^{\prime}}$ for which there are special symmetries, there will be at least two possible epistemic utilities that may result given that $C$ obtains.

Now given an algebra $\mathbf{A}_{i}^{t^{\prime}}$, the cardinality of the class of possible credal functions over $\mathbf{A}_{i}^{t^{\prime}}$ will be equal to $|\mathbb{R}|^{\left|\mathbf{A}_{i}^{t^{\prime}}\right|}$. Since $|\mathbb{R}|^{\left|\mathbf{A}_{i}^{t^{\prime}}\right|}$ is infinite and is greater than $\left|\mathbf{A}_{i}^{t^{\prime}}\right|$, we have that, given any algebra $\mathbf{A}_{i}^{t^{\prime}}$, the cardinality of the class of possible credal functions over $\mathbf{A}_{i}^{t^{\prime}}$ is guaranteed to be infinite and greater than $\left|\mathbf{A}_{i}^{t^{\prime}}\right|$. And so, if $\mathbf{C}_{i}^{t^{\prime}}$ is the set of propositions uniquely characterizing $i$ 's possible credal distributions over $\mathbf{A}_{i}^{t^{\prime}}$, it follows that $\left|\mathbf{C}_{i}^{t^{\prime}}\right|$ will be infinite and will be greater than $\left|\mathbf{A}_{i}^{t^{\prime}}\right|$.

Putting these pieces together, then, given that, in general, $\left|\mathbf{C}_{i}^{t^{\prime}}\right|$ will be infinite and will be greater than $\left|\mathbf{A}_{i}^{t^{\prime}}\right|$, and that, in all but some unusual cases, with the exception of a relatively small, non-cofinite, number of $C \in \mathbf{C}_{i}^{t^{\prime}}$ for which there are special symmetries, there will be at least two possible epistemic utilities that may result given that $C$ obtains, we should expect that, in all but some unusual cases, $\left|\mathbf{C}_{>2}\left(\mathbf{C}_{i}^{t^{\prime}}\right)\right|$ will be infinite and will be such that $\left|\mathbf{C}_{\geq 2}\left(\mathbf{C}_{i}^{t^{\prime}}\right)\right| \geq\left|\mathbf{A}_{i}^{t^{\prime}}\right|$. And so, we have that, in all but some very unusual cases, Bifurcation will hold.

Now let's turn to Exponentiation. In support of the claim that, in all but some very unusual cases, Exponentiation will hold, consider the following modal recombination principle:

Value Recombination: Let $\mathbf{C}_{i}^{t^{\prime}}$ be a set of credal options for $i$ at $t^{\prime}$. Let $\mathbf{B}$ be a set of propositions of the form $C \square \mapsto\left[E V_{i}^{t^{\prime}}=x\right]$, where $C \in \mathbf{C}_{i}^{t^{\prime}}$, and, for each $C \in \mathbf{C}_{i}^{t^{\prime}}$, there is at most one such proposition in $\mathbf{B}$. If, for each $C \square \leftrightarrow\left[E V_{i}^{t^{\prime}}=x\right] \in \mathbf{B}$, there is some $w \in W$ such that $C \square \mapsto\left[E V_{i}^{t^{\prime}}=x\right]$ obtains at $w$, then there is some $w^{\prime} \in W$ such that $\cap \mathbf{B}$ obtains at $w^{\prime}$.

\footnotetext{
${ }^{28}$ Again, this will hold if, in cases in which an agent $i$ at $w$ and $t$ has a credal state defined over a finite algebra, we take $e u(t, i, w)$ to be given by the negative Brier score.
} 
To see why this principle is at least prima facie plausible, recall that $W$ is the set of epistemic possibilities, i.e., maximally specific possibilities that cannot be ruled out a priori. Value Recombination, then, claims that if one cannot rule out a priori any member of some set of propositions of the form $C \square \rightarrow\left[E V_{i}^{t^{\prime}}=x\right]$, where $C \in \mathbf{C}_{i}^{t^{\prime}}$, and there is at most one such proposition in the set for each $C \in \mathbf{C}_{i}^{t^{\prime}}$, then one cannot a priori rule out the conjunction of such propositions. At first glance, at least, this seems to me to be quite plausible. For it's hard to see what sort of a priori discernible connection there might be amongst a class of propositions of the form $C \square \rightarrow\left[E V_{i}^{t^{\prime}}=x\right]$, given that their antecedents are incompatible, that would allow one to rule out the combination of such counterfactuals without ruling out some particular counterfactual.

There is, however, at least some reason to not endorse this principle in an unrestricted form. For assuming that the space of epistemically possible worlds forms a set, it follows that in certain cases it may be that the space of epistemic possibilities is simply not large enough for this recombination principle to be satisfied. ${ }^{29}$ However, it is worth stressing that in order for this to be so, the algebra over which the members $\mathbf{C}_{i}^{t^{\prime}}$ are defined must be extremely fine-grained.

To see this, let $\mathcal{B}$ be the set of sets such that each $\mathbf{B} \in \mathcal{B}$ is such that, for each $C \in \mathbf{C}_{i}^{t^{\prime}}$, there is exactly one proposition of the form $C \square \leftrightarrow\left[E V_{i}^{t^{\prime}}=x\right]$ in $\mathbf{B}$, where $C \square \rightarrow\left[E V_{i}^{t^{\prime}}=x\right]$ holds at some $w$, and nothing else is in $\mathbf{B}$. If, then, the space of epistemically possible worlds $W$ forms a set and is large enough to accommodate the modal recombinations required by the instance of Value Recombination for $\mathbf{C}_{i}^{t^{\prime}}$ it must be that $|W| \geq|\mathcal{B}|$.

Under what conditions, then, will it be the case that $|\mathcal{B}|>|W|$ ? We can determine an upper-bound on the value of $|\mathcal{B}|$ as follows. Suppose that, for each $C \in \mathbf{C}_{i}^{t^{\prime}}$ and each proposition of the form $\left[E V_{i}^{t^{\prime}}=x\right]$, there is some $w$ at which $C \square \rightarrow\left[E V_{i}^{t^{\prime}}=x\right]$ holds. (Now, in fact we shouldn't expect this to be the case. However, since the actual value of $|\mathcal{B}|$ will be at least as great as its value under this supposition, by determining the cardinality of $\mathcal{B}$ under this supposition we can set an upper bound for this value.) Since the cardinality of propositions of the form $\left[E V_{i}^{t^{\prime}}=x\right]$ is $|\mathbb{R}|$, it follows, given our assumption, that $|\mathcal{B}|=|\mathbb{R}|^{\left|\mathbf{C}_{i}^{t^{\prime}}\right|}$. And since, $\left|\mathbf{C}_{i}^{t^{\prime}}\right| \geq|\mathbb{R}|$, we have $|\mathbb{R}|^{\left|\mathbf{C}_{i}^{t^{\prime}}\right|}=2^{\left|\mathbf{C}_{i}^{t^{\prime}}\right|}$. ${ }^{30}$ Thus, given that $\left|\mathbf{C}_{i}^{t^{\prime}}\right|=|\mathbb{R}|^{\left|\mathbf{A}_{i}^{t^{\prime}}\right|}$, we have that $|\mathcal{B}|=2^{|\mathbb{R}|^{\left|\mathbf{A}_{i}^{\prime}\right|}}$. And so, in general, we will have that $|\mathcal{B}| \leq 2^{|\mathbb{R}|^{\left|\mathbf{A}_{i}^{t^{\prime}}\right|}}$.

Now, for present purposes, the key point to note is that, while, on the assumption that $W$ forms a set, there will certainly be algebras $\mathbf{A}_{i}^{t^{\prime}}$ such that $|W|<2^{\mid \mathbb{R}^{\left|\mathbf{A}_{i}^{t^{\prime}}\right|}}$, for any algebra that an actual agent might entertain we should, I think, expect that $2^{\mid \mathbb{R}^{\left|\mathbf{A}_{i}^{t^{\prime}}\right|}}$, and so $|\mathcal{B}|$, will be vastly smaller than $|W|$. We should, then, expect that, in all but some unusual cases, the space of epistemic possibilities will be large enough to accommodate the combinatorial possibilities demanded by Value Recombination. And so, given its prima facie plausibility, I think that we should expect that in typical cases, in which the members $\mathbf{C}_{i}^{t^{\prime}}$ are not defined over extremely fine-grained algebras, Value Recombination will hold.

\footnotetext{
${ }^{29}$ See Chalmers (2011), though, for an argument that the space of epistemically possible worlds is too large to form a set.

${ }^{30}$ In general, if $2 \leq \kappa \leq \lambda$ and $\lambda$ is infinite, then $\kappa^{\lambda}=2^{\lambda}$. See Jech (2000) Lemma 5.6.
} 


\section{Claim: Exponentiation follows from Value Recombination.}

Justification: Here is a sketch of how this result may be proved. We want to show that, given Value Recombination, we have that for each $\mathbf{C} \subseteq \mathbf{C}_{\geq 2}\left(\mathbf{C}_{i}^{t^{\prime}}\right)$, $|\mathbf{E D}(\mathbf{C})| \geq 2^{|\mathbf{C}|}$. Now $2^{|\mathbf{C}|}=|\{f: f: \mathbf{C} \rightarrow\{0,1\}\}|$. To show that, for each $\mathbf{C} \subseteq$ $\mathbf{C}_{\geq 2}\left(\mathbf{C}_{i}^{t^{\prime}}\right),|\mathbf{E D}(\mathbf{C})| \geq 2^{|\mathbf{C}|}$, it suffices then to show that, for each $\mathbf{C} \subseteq \mathbf{C}_{\geq 2}\left(\mathbf{C}_{i}^{t^{\prime}}\right)$, there is an injective function $g:\{f: f: \mathbf{C} \rightarrow\{0,1\}\} \rightarrow \mathbf{E D}(\mathbf{C})$.

Let $\mathbf{C}$ be an arbitrary subset of $\mathbf{C}_{\geq 2}\left(\mathbf{C}_{i}^{t^{\prime}}\right)$. To show that there is such a function for $\mathbf{C}$, let $r(\cdot): \mathbf{C} \rightarrow \cup\{\mathbf{E D}(\{C\}): C \in \mathbf{C}\} \times \cup\{\mathbf{E D}(\{C\}): C \in \mathbf{C}\}$ be a function such that $r(C)=\left\langle C \square \rightarrow\left[E V_{i}^{t^{\prime}}=x\right], C \square \mapsto\left[E V_{i}^{t^{\prime}}=y\right]\right\rangle$, where $C \square \mapsto\left[E V_{i}^{t^{\prime}}=\right.$ $x], C \square \leftrightarrow\left[E V_{i}^{t^{\prime}}=y\right] \in \mathbf{E D}(\{C\})$. We let $\mathbf{R}=\{r(C): C \in \mathbf{C}\}$. Let $\mathbf{Q}$ be the set of functions mapping elements of $\mathbf{R}$ to elements of $\cup\{\mathbf{E D}(\{C\}): C \in \mathbf{C}\}$ that satisfy the condition that, for each $R \in \mathbf{R}, q(R) \in R$. Given Value Recombination, we have that for each $q \in \mathbf{Q}$, there is a non-empty set $P_{q}=\cap\{q(r(C)): C \in \mathbf{C}\}$. Let $\mathbf{S}=\left\{P_{q}: q \in \mathbf{Q}\right\}$.

Now there is clearly a bijection from $\{f: f: \mathbf{C} \rightarrow\{0,1\}\}$ to $\mathbf{Q}$. And so there is a bijection from $\{f: f: \mathbf{C} \rightarrow\{0,1\}\}$ to $\mathbf{S}$. Let us pick an arbitrary bijection of this sort and call it $h$.

For each $S \in \mathbf{S}$, there will be a unique $\mathbf{D}_{S} \subseteq \mathbf{E D}(\mathbf{C})$ such that $\cup \mathbf{D}_{S}=S$. Moreover, for each $S, S^{\prime} \in \mathbf{S}, \mathbf{D}_{S} \cap \mathbf{D}_{S^{\prime}}=\emptyset$. Let $m: \mathbf{S} \rightarrow \mathbf{E D}(\mathbf{C})$ be such that $m(S) \subseteq S$. Given that, for each $S, S^{\prime} \in \mathbf{S}, \mathbf{D}_{S} \cap \mathbf{D}_{S^{\prime}}=\emptyset$, this function will be injective, i.e., one-to-one.

Now we let $f=m \circ h$. This function will be an injection from $\{f: f: \mathbf{C} \rightarrow\{0,1\}\}$ to $\mathbf{E D}(\mathbf{C})$.

Since Exponentiation follows from Value Recombination, and since we should expect that, in all but some unusual cases, Value Recombination holds, we should expect, then, that, in all but some unusual cases, Exponentiation holds. And, given our previous arguments, we have that, in all but some unusual cases, Bifurcation holds. Furthermore, since the situations in which either of these principles might fail are, I think, highly unusual, we should, in addition expect that, in all but some unusual cases, both Bifurcation and Value Recombination will hold.

We can now show that, given that both Bifurcation and Value Recombination hold in all but some unusual cases, it follows that, if $\mathbf{C}_{i}^{t^{\prime}}$ is the set of propositions uniquely characterizing $i$ 's possible credal distributions over $\mathbf{A}_{i}^{t^{\prime}}$, then, in all but some very usual cases, there will be some infinite $\mathbf{C} \subseteq \mathbf{C}_{i}^{t^{\prime}}$ such that, for each $C \in \mathbf{C}$, there is some $A \in \mathbf{A t}_{i}^{t^{\prime}}$, for which $E U(C \cap A)$ is undefined, i.e., in all but some unusual cases Undefinedness holds.

Claim: Bifurcation and Exponentiation entail Undefinedness.

Justification: To see this, first note that, if $\mathbf{C} \subseteq \mathbf{C}_{i}^{t^{\prime}}$, and, for every $C \in \mathbf{C}$ and every $A \in \mathbf{A} \mathbf{t}_{i}^{t^{\prime}}, E U(C \cap A)$ is well-defined, then either $\mathbf{A} \mathbf{t}_{i}^{t^{\prime}}=\mathbf{E D}(\mathbf{C})$, or $\mathbf{A} \mathbf{t}_{i}^{t^{\prime}}$ is 
a fine-graining of $\mathbf{E D}(\mathbf{C})$. It follows that if $\mathbf{C} \subseteq \mathbf{C}_{i}^{t^{\prime}}$, and, for every $C \in \mathbf{C}$ and every $A \in \mathbf{A t}_{i}^{t^{\prime}}, E U(C \cap A)$ is well-defined, then $\left|\mathbf{A t}_{i}^{t^{\prime}}\right| \geq|\mathbf{E D}(\mathbf{C})|$.

Now suppose that $\left|\mathbf{C}_{\geq 2}\left(\mathbf{C}_{i}^{t^{\prime}}\right)\right|$ is infinite and is such that $\left|\mathbf{C}_{\geq 2}\left(\mathbf{C}_{i}^{t^{\prime}}\right)\right| \geq\left|\mathbf{A}_{i}^{t^{\prime}}\right|$. We'll show that, given this assumption, it follows from Exponentiation, that there will be some infinite $\mathbf{C} \subseteq \mathbf{C}_{i}^{t^{\prime}}$ such that, for each $C \in \mathbf{C}$, there is some $A \in \mathbf{A t}_{i}^{t^{\prime}}$, for which $E U(C \cap A)$ is undefined.

Let $\mathbf{C}^{\prime} \subseteq \mathbf{C}_{\geq 2}\left(\mathbf{C}_{i}^{t^{\prime}}\right)$ be the set of $C \in \mathbf{C}_{\geq 2}\left(\mathbf{C}_{i}^{t^{\prime}}\right)$ such that $E U(C \cap A)$ is welldefined, for every $A \in \mathbf{A t}_{i}^{t^{\prime}}$. Then we have that $\left|\mathbf{A t}_{i}^{t^{\prime}}\right| \geq\left|\mathbf{E D}\left(\mathbf{C}^{\prime}\right)\right|$. And, by Exponentiation, we have that $\left|\mathbf{E D}\left(\mathbf{C}^{\prime}\right)\right| \geq 2^{\left|\mathbf{C}^{\prime}\right|}$. And so we have $\left|\mathbf{C}_{\geq 2}\left(\mathbf{C}_{i}^{t^{\prime}}\right)\right| \geq$ $\left|\mathbf{A t}_{i}^{t^{\prime}}\right| \geq 2^{\left|\mathbf{C}^{\prime}\right|}$. In general, though, if $|\Sigma|$ is infinite and $\Delta \subseteq \Sigma$ is such that $2^{|\Delta|} \leq|\Sigma|$, then $|\Sigma-\Delta|$ is infinite. If, then, we let $\mathbf{C}=\mathbf{C}_{\geq 2}\left(\mathbf{C}_{i}^{t^{\prime}}\right)-\mathbf{C}^{\prime}$, it follows that $|\mathbf{C}|$ is infinite. But, by definition, we have that for each $C \in \mathbf{C}$, there is some $A \in \mathbf{A t}_{i}^{t^{\prime}}$, such that $E U(C \cap A)$ is undefined.

Given, then, that $\left|\mathbf{C}_{\geq 2}\left(\mathbf{C}_{i}^{t^{\prime}}\right)\right|$ is infinite and is such that $\left|\mathbf{C}_{\geq 2}\left(\mathbf{C}_{i}^{t^{\prime}}\right)\right| \geq\left|\mathbf{A}_{i}^{t^{\prime}}\right|$, it follows, given Exponentiation, that there will be some infinite $\mathbf{C} \subseteq \mathbf{C}_{i}^{t^{\prime}}$ such that, for each $C \in \mathbf{C}$, there is some $A \in \mathbf{A t}_{i}^{t^{\prime}}$, for which $E U(C \cap A)$ is undefined.

Given Bifurcation, though, if $\mathbf{C}_{i}^{t^{\prime}}$ is the set of propositions uniquely characterizing $i$ 's possible credal distributions over $\mathbf{A}_{i}^{t^{\prime}}$, then $\left|\mathbf{C}_{\geq 2}\left(\mathbf{C}_{i}^{t^{\prime}}\right)\right|$ will be infinite and will be such that $\left|\mathbf{C}_{\geq 2}\left(\mathbf{C}_{i}^{t^{\prime}}\right)\right| \geq\left|\mathbf{A}_{i}^{t^{\prime}}\right|$. It follows, then, given Exponentiation and Bifurcation, that if $\mathbf{C}_{i}^{t^{\prime}}$ is the set of propositions uniquely characterizing $i$ 's possible credal distributions over $\mathbf{A}_{i}^{t^{\prime}}$, then there will be some infinite $\mathbf{C} \subseteq \mathbf{C}_{i}^{t^{\prime}}$ such that, for each $C \in \mathbf{C}$, there is some $A \in \mathbf{A} \mathbf{t}_{i}^{t^{\prime}}$, for which $E U(C \cap A)$ is undefined. And so we have Undefinedness.

Given, then, that both Bifurcation and Exponentiation hold in all but some unusual cases, we have that if $\mathbf{C}_{i}^{t^{\prime}}$ is the set of propositions uniquely characterizing $i$ 's possible credal distributions over $\mathbf{A}_{i}^{t^{\prime}}$, then, in all but some very unusual cases, there will be an infinite $\mathbf{C} \subseteq \mathbf{C}_{i}^{t^{\prime}}$ such that, for each $t$ in the stable interval leading up to $t^{\prime}, E G_{i}^{t}(C)$ will be undefined for each $C \in \mathbf{C}$.

In $\S 1$, we noted that if $G_{i}^{t}(O)$ is not well-defined but $E C U_{i}^{t}(O)$ is, then $\operatorname{Cr}_{i}^{t}(\cdot)$ must be quasi-opinionated about $\mathbf{D}\left(\{O\}, u_{i}^{t}\right)$, i.e., the agent must be certain that the utility value that would obtain were $O$ to be realized is amongst some proper subset of the set of a priori epistemically possible utility values that could be realized were $O$ to obtain. The same principle applies in cases in which $E G_{i}^{t}(C)$ is not well-defined but $E E C U_{i}^{t}(O)$ is. In such cases, $i$ must have a credal state $C r_{i}^{t}(\cdot)$ that is quasi-opinionated about $\operatorname{ED}(\{C\})$, so that $i$ is certain that the epistemic utility value that would obtain were $C$ to be realized is amongst some proper subset of the set of a priori epistemically possible epistemic utility values that could be realized were $C$ to obtain.

Given, then, that Undefinedness holds, in all but some unusual cases, we have that, in all but some unusual cases, if, in the stable interval leading up to $t^{\prime}, i$ is not quasi-opinionated about $\operatorname{ED}(\{C\})$, for an infinite number of $C \in \mathbf{C}_{i}^{t^{\prime}}$, then $E E C U_{i}(\cdot)$ will not be well-defined for an infinite $\mathbf{C}^{U} \subseteq \mathbf{C}_{i}^{t^{\prime}}$. Given this, though, it follows from No Credal Distinctions that, 
in all but some very unusual cases, if, in the stable interval leading up to $t^{\prime}, i$ is not quasiopinionated about $\mathbf{E D}(\{C\})$, for an infinite number of $C \in \mathbf{C}_{i}^{t^{\prime}}$, then no non-trivial deontic distinctions can be drawn amongst an agent's credal options. And so we have that, in all but some unusual cases, Opinionation holds.

This strikes me as a very bad result for credal consequentialism. To appreciate why this is so, it's worth stepping back and considering the general picture of rationality that a credal consequentialist theory provides.

The principle that captures the core thought behind credal consequentialism, Credal Maximization, is a diachronic principle of rationality. This principle tells us what sort of credal state it is rationally permissible or obligatory for an agent to have at a time $t^{\prime}$ given certain facts about the agent's credal state prior to $t^{\prime}$. And the same is true of the other principles of credal consequentialism that we've considered, such as Global Credal Dominance Permissivism. These principles impose certain constraints on which patterns of credal states over time count as rationally permissible and which count as rationally impermissible.

It's natural to think that such diachronic principles should be supplemented by certain boundary conditions. Let us call the credal state that an agent has at the beginning of their epistemic life their initial credal state. It's natural, then, to think that the diachronic principles of rationality provided by credal consequentialism should be supplemented by principles that tell us which initial credal states are rationally permissible and which are rationally impermissible.

Now one prima facie plausible constraint on such boundary conditions is:

Dependence Hypothesis Neutrality: If an agent $i$ has an initial credal state at some time $t$, then, for any $t^{\prime}>t$, it is rationally permissible for $i$ 's credal state to be such that, for each $C \in \mathbf{C}_{i}^{t^{\prime}}, i$ is not certain that the epistemic utility value that would obtain were $C$ to be realized is amongst some proper subset of the set of a priori epistemically possible epistemic utility values that could be realized were $C$ to obtain.

This claim follows from a very plausible general principle that says that an agent should not be rationally required have an initial credal state that makes them certain of some proper subset of the set of a priori epistemic possibilities. ${ }^{31}$

Given this plausible principle, though, we can see that the picture of credal rationality that results from credal consequentialism has some deeply implausible consequences.

Let $t$ be the time at which $i$ has their initial credal state. Now, given Dependence Hypothesis Neutrality and the dynamic principles of rationality endorsed by the proponent of credal consequentialism, for any algebra $\mathbf{A}_{i}^{t^{\prime}}$, it should, in general, be possible for an agent to have an initial credal state at $t, C r(\cdot)_{i}^{t}$, defined over this algebra such that there is some time $t^{\prime}>t$, for which the following two conditions obtain. First, given $\operatorname{Cr}(\cdot)_{i}^{t}$, for each $C \in \mathbf{C}_{i}^{t^{\prime}}$,

\footnotetext{
${ }^{31}$ Note that if the space of epistemic possibilities for an agent is uncountable in size, then it's certainly true that the agent will not be able to assign positive credence to each such possibility. What this general principle requires in such cases, though, is simply that the agent's credal density function be spread out over the whole space of epistemic possibilities and not restricted to some proper subspace.
} 
$i$ is not certain that the epistemic utility value that would obtain were $C$ to be realized is amongst some proper subset of the set of a priori epistemically possible epistemic utility values that could be realized were $C$ to obtain. Second, it is rationally permissible for $i$ to maintain $\operatorname{Cr}(\cdot)_{i}^{t}$ as their credal state through the half-closed interval $\left[t, t^{\prime}\right)$.

To see this, note first that, given Dependence Hypothesis Neutrality, $i$ need not be irrational in virtue of $\operatorname{Cr}(\cdot)_{i}^{t}$ satisfying the first condition. Second, note that the diachronic principles of rationality endorsed by the proponent of credal consequentialism, such as Credal Maximization and Global Credal Dominance Permissivism, impose no constraints on $i$ 's credal state for any time $t^{\prime \prime}<t^{\prime}$, given the assumption that $i$ 's credal state satisfies the first condition. And so the diachronic principles of rationality endorsed by the proponent of credal consequentialism do not rule out $\operatorname{Cr}(\cdot)_{i}^{t}$ satisfying the second condition, given that it satisfies the first. Of course, one might impose further conditions to rule out such cases. But it's hard to see what conditions one might appeal to here that would serve to rule out the rationality of maintaining one's initial credences in these sorts of cases that wouldn't serve to, in general, rule out the rationality of an agent maintaining their credal state over any positive interval of time. But the proponent of credal consequentialism should surely reject any such general prohibition. For principles such as Credal Maximization and Global Credal Dominance Permissivism require such stable credal states to issue any verdicts at all. The proponent of credal consequentialism, then, should allow, given Dependence Hypothesis Regularity, that, for any algebra $\mathbf{A}_{i}^{t^{\prime}}$, an agent may have a credal state $\operatorname{Cr}(\cdot)_{i}^{t}$ satisfying both of the above conditions.

We've seen, though, that, in all but a few cases, if an agent $i$ has stable credences leading up to some time $t^{\prime}$ and $i$ is not quasi-opinionated about $\mathbf{E D}(\{C\})$, for an infinite number of $C \in \mathbf{C}_{i}^{t^{\prime}}$, then, either, for each $C \in \mathbf{C}_{i}^{t^{\prime}}$, it is rationally permissible for $i$ to realize $C$, or, for each $C \in \mathbf{C}_{i}^{t^{\prime}}$, it is rationally impermissible for $i$ to realize $C$, or, for each $C \in \mathbf{C}_{i}^{t^{\prime}}$, it is neither rationally permissible nor rationally impermissible for $i$ to realize $C$.

It follows, then, that for almost any algebra $\mathbf{A}_{i}^{t^{\prime}}$ and agent $i$, there are possible situations in which, for some time $t^{\prime}$, either (a) the agent starts out with rational credences defined over $\mathbf{A}_{i}^{t^{\prime}}$ and at $t^{\prime}$ the agent may rationally adopt any possible credal state defined over $\mathbf{A}_{i}^{t^{\prime}}$, or (b) the agent starts out with rational credences defined over $\mathbf{A}_{i}^{t^{\prime}}$ and at $t^{\prime}$ the agent is rationally prohibited from adopting any credal state defined over $\mathbf{A}_{i}^{t^{\prime}}$, or (c) the agent starts out with rational credences defined over $\mathbf{A}_{i}^{t^{\prime}}$ and at $t^{\prime}$ any credal state defined over $\mathbf{A}_{i}^{t^{\prime}}$ that the agent may adopt has no deontic status attaching to it.

Each of these options seems to me to lead to a deeply distorted picture of credal rationality. Option (a) leads to an overly permissive picture of credal rationality. According to this option, for almost every algebra $\mathbf{A}_{i}^{t^{\prime}}$ and agent $i$, there are rational paths that allow an agent to adopt any credences they like no matter how bizarre. Option (b) leads to an overly prohibitive picture of credal rationality. According to this option, for almost every algebra $\mathbf{A}_{i}^{t^{\prime}}$ and agent $i$, there are rational dead-ends, whereby, given no antecedent irrationality, an agent is left with no rational options available to them. Finally, option (c) leads to a overly reticent picture of credal rationality. According to this option, for almost every algebra $\mathbf{A}_{i}^{t^{\prime}}$ and agent $i$, there are gappy paths whereby a rational initial segment is guaranteed to lead 
to a point that simply cannot be classified as rationally permissible or impermissible.

None of these claims seems to me to be remotely plausible. We have, then, reason to be quite skeptical of the picture credal rationality that results given the commitments of credal consequentialism.

\section{Two Responses}

I've argued that, given some plausible assumptions, credal consequentialism leads to a highly unattractive picture of credal rationality. I now want to consider two ways of resisting this argument.

\subsection{Coarse-Grained Credal Options}

A key assumption in the preceding argument is that $\mathbf{C}_{i}^{t^{\prime}}$-i.e., the set of propositions characterizing $i$ 's credal options - is the set of propositions uniquely characterizing $i$ 's possible credal distributions over $\mathbf{A}_{i}^{t^{\prime}}$. For the preceding argument relied on the claim that, in typical cases, $\left|\mathbf{C}_{\geq 2}\left(\mathbf{C}_{i}^{t^{\prime}}\right)\right|$ will be infinite and will be such that $\left|\mathbf{C}_{\geq 2}\left(\mathbf{C}_{i}^{t^{\prime}}\right)\right| \geq\left|\mathbf{A}_{i}^{t^{\prime}}\right|$. And this claim is plausible given that $\mathbf{C}_{i}^{t^{\prime}}$ is the set of propositions uniquely characterizing $i$ 's possible credal distributions over $\mathbf{A}_{i}^{t^{\prime}}$. For, as we noted, the set of possible credal distributions over $\mathbf{A}_{i}^{t^{\prime}}$ will have a cardinality equal to $\mid \mathbb{R}^{\left|\mathbf{A}_{i}^{t^{\prime}}\right|}$, and so will be guaranteed to be infinite and greater than the cardinality of $\mathbf{A}_{i}^{t^{\prime}}$. And, in typical cases, we should expect $\mathbf{C}_{\geq 2}\left(\mathbf{C}_{i}^{t^{\prime}}\right)$ to contain all but a small, non-cofinite, subset of $\mathbf{C}_{i}^{t^{\prime}}$.

If, however, we take the members of $\mathbf{C}_{i}^{t^{\prime}}$ to be unions of propositions uniquely characterizing $i$ 's possible credal distributions over $\mathbf{A}_{i}^{t^{\prime}}$, then, at least in principle, we may restrict the size of $\mathbf{C}_{i}^{t^{\prime}}$ so that it isn't the case that $\left|\mathbf{C}_{\geq 2}\left(\mathbf{C}_{i}^{t^{\prime}}\right)\right|$ is infinite and greater than $\left|\mathbf{A}_{i}^{t^{\prime}}\right|$. In this way, then, one can block the preceding argument for the claim that there will be some infinite $\mathbf{C} \subseteq \mathbf{C}_{i}^{t^{\prime}}$ such that, for each $C \in \mathbf{C}$, there is some $A \in \mathbf{A t}_{i}^{t^{\prime}}$, for which $E U(C \cap A)$ is undefined. Indeed, if the cardinality of $\mathbf{C}_{i}^{t^{\prime}}$ is appropriately restricted, then it is easy to show that, in certain cases, the cardinality of $\mathbf{A}_{i}^{t^{\prime}}$ may be large enough to allow that $E U(C \cap A)$ is well-defined, for each $C \in \mathbf{C}_{i}^{t^{\prime}}$ and each $A \in \mathbf{A} \mathbf{t}_{i}^{t^{\prime}} \cdot{ }^{32}$

Let's consider, then, the possibility that $\mathbf{C}_{i}^{t^{\prime}}$ may be a set of unions of propositions that uniquely characterize $i$ 's possible credal distributions over $\mathbf{A}_{i}^{t^{\prime}}$. I'll argue that, while this way of developing a credal consequentialist account may allow one to block the preceding argument, it also has some undesirable consequences.

Let $\mathbf{C}$ be a set of credal options in a putative credal decision problem. We'll call a set of unions of the members of $\mathbf{C}$ a set of coarse-grainings of $\mathbf{C}$, and we'll call $\mathbf{C}$ a set of fine-grainings of this set of unions.

\footnotetext{
${ }^{32}$ Here's a simple way of seeing this. Suppose that we take there to be two members of $\mathbf{C}_{i}^{t^{\prime}}$. Call these $C_{1}$ and $C_{2}$. Given that we are taking epistemic utility values to be real numbers, it follows that $\left|\mathbf{E D}\left(\left\{C_{1}\right\}\right)\right| \leq|\mathbb{R}|$ and $\left|\mathbf{E D}\left(\left\{C_{2}\right\}\right)\right| \leq|\mathbb{R}|$. But, then, it follows that $\left|\mathbf{E D}\left(\mathbf{C}_{i}^{t^{\prime}}\right)\right| \leq\left|\mathbf{E D}\left(\left\{C_{1}\right\}\right)\right| \times\left|\mathbf{E D}\left(\left\{C_{2}\right\}\right)\right| \leq|\mathbb{R}|$. Thus, as long as $\left|\mathbf{A} \mathbf{t}_{i}^{t^{\prime}}\right| \geq|\mathbb{R}|$, it is at least in principle possible that, for each $C \in \mathbf{C}_{i}^{t^{\prime}}$ and each $A \in \mathbf{A t}_{i}^{t^{\prime}}, E U(C \cap A)$ is well-defined.
} 
Given a putative credal decision problem with a set of options $\mathbf{C}$, we can construct an alternative credal decision problem involving a coarse-graining of this set of options To see this, consider the following toy model.

Fine-Grained Decision: Let $\mathbf{C}=\left\{C_{1}, C_{2}, C_{3}, C_{4}\right\}$. And we let $\mathbf{E D}(\mathbf{C})=$ $\left\{D_{1}, D_{2}\right\}$ where $D_{1}=\left[C_{1} \square \hookrightarrow\left[E V_{i}^{t^{\prime}}=5\right]\right] \cap\left[C_{2} \square \leftrightarrow\left[E V_{i}^{t^{\prime}}=3\right]\right] \cap\left[C_{3} \square \leftrightarrow\right.$ $\left.\left[E V_{i}^{t^{\prime}}=1\right]\right] \cap\left[C_{4} \square \leftrightarrow\left[E V_{i}^{t^{\prime}}=7\right]\right]$, and $D_{2}=\left[C_{1} \square \leftrightarrow\left[E V_{i}^{t^{\prime}}=6\right]\right] \cap\left[C_{2} \square \rightarrow\left[E V_{i}^{t^{\prime}}=\right.\right.$ $4]] \cap\left[C_{3} \square \leftrightarrow\left[E V_{i}^{t^{\prime}}=2\right]\right] \cap\left[C_{4} \square \leftrightarrow\left[E V_{i}^{t^{\prime}}=8\right]\right]$.

We can represent this as follows:

\begin{tabular}{|l|c|c|}
\hline & $D_{1}$ & $D_{2}$ \\
\hline$C_{1}$ & 5 & 6 \\
\hline$C_{2}$ & 3 & 4 \\
\hline$C_{3}$ & 1 & 2 \\
\hline$C_{4}$ & 7 & 8 \\
\hline
\end{tabular}

Now, suppose that one claims that the credal options available to $i$ at $t^{\prime}$ are really coarsegrainings of $\mathbf{C}$ and that, in particular, $\mathbf{C}_{i}^{t^{\prime}}=\left\{C_{1} \cup C_{2}, C_{3} \cup C_{4}\right\}$. There is a straightforward way of taking the preceding credal decision problem and converting it into a credal decision problem for this new coarse-grained set of options. To do this, we divide up each of $D_{1}$ and $D_{2}$ into disjoint subspaces that determine which of the options $C_{1}$ and $C_{2}$ the agent would realize were they to realize $C_{1} \cup C_{2}$ and which of the options $C_{3}$ and $C_{4}$ the agent would realize were they to realize $C_{3} \cup C_{4}$.

Coarse-Grained Decision: Let $S_{1}=\left[C_{1} \cup C_{2} \square \rightarrow C_{1}\right] \cap\left[C_{3} \cup C_{4} \square \rightarrow C_{3}\right], S_{2}=$ $\left[C_{1} \cup C_{2} \square \rightarrow C_{1}\right] \cap\left[C_{3} \cup C_{4} \square \rightarrow C_{4}\right], S_{3}=\left[C_{1} \cup C_{2} \square \rightarrow C_{2}\right] \cap\left[C_{3} \cup C_{4} \square \rightarrow C_{3}\right]$, $S_{4}=\left[C_{1} \cup C_{2} \square \rightarrow C_{2}\right] \cap\left[C_{3} \cup C_{4} \square \rightarrow C_{4}\right]$. Then we can let $\mathbf{E D}\left(\mathbf{C}_{i}^{t^{\prime}}\right)=\left\{D_{1} \cap\right.$ $\left.S_{1}, D_{1} \cap S_{2}, D_{1} \cap S_{3}, D_{1} \cap S_{4}, D_{2} \cap S_{1}, D_{2} \cap S_{2}, D_{2} \cap S_{3}, D_{2} \cap S_{4}\right\}$.

We can now represent the new credal decision problem for the coarse-grained set of options as follows:

\begin{tabular}{|c|c|c|c|c|c|c|c|c|}
\hline & $D_{1} \cap S_{1}$ & $D_{1} \cap S_{2}$ & $D_{1} \cap S_{3}$ & $D_{1} \cap S_{4}$ & $D_{2} \cap S_{1}$ & $D_{2} \cap S_{2}$ & $D_{2} \cap S_{3}$ & $D_{2} \cap S_{4}$ \\
\hline$C_{1} \cup C_{2}$ & 5 & 5 & 3 & 3 & 6 & 6 & 4 & 4 \\
\hline$C_{3} \cup C_{4}$ & 1 & 7 & 1 & 7 & 2 & 8 & 2 & 8 \\
\hline
\end{tabular}

It's important to note that these two credal decision problems are not mere notational variants. Indeed, it may be that, given the second sort of credal decision problem, the coarse-grained option that maximizes expected epistemic utility will not include the finegrained option that maximizes expected epistemic utility, given the first sort of credal decision problem. ${ }^{33}$

\footnotetext{
${ }^{33}$ To see this consider the two toy credal decision problems outlined above. Since $C_{4}$ dominates all other
} 
The key fact here is that in converting a credal decision problem involving fine-grained options into one involving coarse-grained options, one must take it that which of the finegrained options the agent would realize were they to realize one of the coarse-grained options is an exogenous factor about which the agent may have credences but over which the agent has no control, and which serves to partly characterize the epistemic dependence hypotheses for the coarse-grained options.

Now it's a subtle and difficult question in what sense exactly it is within an agent's power to realize their credal options. If, though, one wants to maintain that an agent's credal options should be thought of as unions of propositions that uniquely characterize $i$ 's possible credal distributions over $\mathbf{A}_{i}^{t^{\prime}}$, then there must be some normatively relevant sense in which it may be within an agent's powers to realize which of these unions obtains, but not within their power to realize which of the particular propositions that uniquely characterizes their credal distribution obtains.

It is, I think, far from obvious that there is any clear sense in which an agent's credal options are within their power that would allow us to draw a principled distinction here. But let us suppose that such a distinction can be drawn and so $\mathbf{C}_{i}^{t^{\prime}}$ is a set of unions of propositions that uniquely characterize $i$ 's possible credal distributions over $\mathbf{A}_{i}^{t^{\prime}}$. I'll now argue that, even given this assumption, credal consequentialism has undesirable consequences.

Assuming that $\mathbf{C}_{i}^{t^{\prime}}$ is a set of unions of propositions that uniquely characterize $i$ 's possible credal distributions over $\mathbf{A}_{i}^{t^{\prime}}$, a credal consequentialist account will, in the first instance, result in verdicts concerning which coarse-grained credal options are rationally permissible or impermissible to realize. Given such deontic verdicts, though, it is natural to ask whether one can extract deontic verdicts concerning the more fine-grained space of propositions uniquely characterizing $i$ 's possible credal distributions over $\mathbf{A}_{i}^{t^{\prime}}$. I'll argue that the answer to this question is 'no'.

To this end, let me begin by considering a more specific question. Let $\mathbf{C}^{P} \subseteq \mathbf{C}_{i}^{t^{\prime}}$ be the set of coarse-grained credal options that it is rationally permissible for the agent to realize. And let $\mathbf{F}^{P}$ be the set of propositions uniquely characterizing $i$ 's possible credal distributions over $\mathbf{A}_{i}^{t^{\prime}}$ that fine-grain the members of $\mathbf{C}^{P}$. Let us, then, begin by focusing on the question of whether, given the permissibility of the members of $\mathbf{C}^{P}$, we can extract deontic verdicts concerning the members of $\mathbf{F}^{P}$. I'll argue that the answer to this question is 'no'.

First, note that if we are to extract deontic verdicts for the members of $\mathbf{F}^{P}$ from the deontic status of the members of $\mathbf{C}^{P}$, then it would seem that the only principled option is to treat all of the members of $\mathbf{F}^{P}$ as having the same deontic status. Thus, if we are to extract deontic verdicts for the members of $\mathbf{F}^{P}$ from the deontic status of the members of $\mathbf{C}^{P}$, then either all of the members of $\mathbf{F}^{P}$ must be rationally permissible to realize, or all of the members of $\mathbf{F}^{P}$ must be rationally impermissible to realize. However, given that $\mathbf{C}^{P}$ is the set of rationally permissible credal options, and that if one realizes some member of $\mathbf{C}^{P}$, then one must realize some member of $\mathbf{F}^{P}$, we should not maintain that all of the members

options it is guaranteed to have the highest expected epistemic utility given the fine-grained credal decision problem. However, since $C_{3}$ is dominated by all the other options, if $i$ gives sufficient credence to $S_{1} \cup S_{3}$, i.e., to the claim that were $C_{3}$ or $C_{4}$ to be realized, $C_{3}$ would be realized, then, given the coarse-grained credal decision problem, $C_{1} \cup C_{2}$ may maximize expected epistemic utility. 
of $\mathbf{F}^{P}$ are rationally impermissible to realize. If, then, we are to extract deontic verdicts for the members of $\mathbf{F}^{P}$ from the deontic statuses of the members of $\mathbf{C}^{P}$, it must be that all of the members of $\mathbf{F}^{P}$ are rationally permissible to realize.

There is, however, good reason to reject the claim that, given that each of the members of $\mathbf{C}^{P}$ is rationally permissible to realize, each of the members of $\mathbf{F}^{P}$ is rationally permissible to realize. And so, given this, there is good reason to think that we cannot extract deontic verdicts for the members of $\mathbf{F}^{P}$ from the deontic statuses of the members of $\mathbf{C}^{P}$.

The problem with maintaining that each of the members of $\mathbf{F}^{P}$ is rationally permissible to realize, given that each of the members of $\mathbf{C}^{P}$ is rationally permissible to realize, is that this commits us, in certain cases, to claiming that some $F \in \mathbf{F}^{P}$ is rationally permissible, at least in part, in virtue of the fact that $i$ takes it to be sufficiently likely that were they to realize some coarse-grained credal option $C=F \cup \ldots$. , they would not realize $F$, but would instead realize some other option that would result in greater epistemic utility.

To see this, suppose, as the account under consideration maintains, that the members of $\mathbf{F}^{P}$ are rationally permissible in virtue of the fact that each of the members of $\mathbf{C}^{P}$ is rationally permissible and the members of $\mathbf{F}^{P}$ are the fine-grainings of these permissible coarse-grained options. Now, in standard cases, the members of $\mathbf{C}^{P}$ will be rationally permissible in virtue of the fact that their expected epistemic utility is at least as great as any other options. But note that, amongst the fine-grainings of some $C \in \mathbf{C}^{P}$, there may be credal options that do very poorly with respect to epistemic utility in various circumstances. Nonetheless, it may be that $C$ has high expected epistemic utility because $i$ expects that were they to realize $C$ they would not realize $F$, but would instead realize some other option that results in greater epistemic utility. ${ }^{34}$ Since, then, $C$ has high expected epistemic utility in virtue of the fact that $i$ expects that were they to realize $C$ they would not realize $F$, and since $C$ is rationally permissible, at least in part, in virtue of the fact that it has sufficiently high expected epistemic utility, if we, in addition, say that $F$ is rationally permissible in virtue of the fact that $C$ is rationally permissible, we seem to be forced to say that $F$ is rationally permissible, at least in part, in virtue of the fact that $i$ expects that were they to realize $C$ they would not realize $F$, but would instead realize some epistemically superior option.

But surely taking it to be likely that were one to realize $C$ one would not realize $F$ but instead would realize some epistemically superior option can't be the sort of fact that serves, even in part, to rationalize $F$. I think, then, that this shows that we can't, in any plausible manner, extract verdicts about the deontic status of the members of $\mathbf{F}^{P}$, given the permissibility of the members of $\mathbf{C}^{P}$, or more generally, given the deontic status of the agent's coarse-grained options.

This, of course, leaves open the question about whether we can extract verdicts concerning the deontic status of those fine-grained credal options that are not members of $\mathbf{F}^{P}$, given verdicts about the deontic status of various coarse-grained options. Similar considerations,

\footnotetext{
${ }^{34} \mathrm{As}$ an example of this consider the toy credal decision problem outlined earlier in this section. In this case, $C_{3}$ results in worse epistemic utility than all of the other fine-grained credal options. Nonetheless, since $C_{4}$ results in greater epistemic utility than all of the other fine-grained credal options, the coarse-grained option $C_{3} \cup C_{4}$ could maximize expected epistemic utility if $i$ assigns sufficiently low credence to $S_{1} \cup S_{3}$, i.e., to the claim that were $C_{3}$ or $C_{4}$ to be realized, $C_{3}$ would be realized.
} 
though, may be used to argue that the answer to this question is also 'no'.

Let $\mathbf{C}^{I}=\mathbf{C}_{i}^{t^{\prime}}-\mathbf{C}^{P}$ and let $\mathbf{F}^{I}$ be the set of propositions uniquely characterizing $i$ 's possible credal distributions over $\mathbf{A}_{i}^{t^{\prime}}$ that fine-grain the members of $\mathbf{C}^{I} \cdot \mathbf{C}^{I}$, then, is the set of coarse-grained credal options that would be rationally impermissible for $i$ to realize, and $\mathbf{F}^{I}$ are the fine-grainings of this set of options.

If we are to extract deontic verdicts for the members of $\mathbf{F}^{I}$ from the deontic status of the members of $\mathbf{C}^{I}$, it would seem that the only principled option is to treat all of the members of $\mathbf{F}^{I}$ as having the same deontic status. If, then, we are to extract deontic verdicts for the members of $\mathbf{F}^{I}$ from the deontic status of the members of $\mathbf{C}^{I}$, then either all of the members of $\mathbf{F}^{I}$ must be rationally permissible to realize, or all of the members of $\mathbf{F}^{I}$ must be rationally impermissible to realize. However, given that $\mathbf{C}^{I}$ is the set of rationally impermissible credal options, and that if one realizes some member of $\mathbf{C}^{I}$, then one must realize some member of $\mathbf{F}^{I}$, we should not maintain that all of the members of $\mathbf{F}^{I}$ are rationally permissible to realize. If, then, we are to extract deontic verdicts for the members of $\mathbf{F}^{I}$ from the deontic status of the members of $\mathbf{C}^{I}$, then it must be that all of the members of $\mathbf{F}^{I}$ are rationally impermissible to realize.

There is, however, good reason to reject the claim that, given that each of the members of $\mathbf{C}^{I}$ is rationally impermissible to realize, each of the members of $\mathbf{F}^{I}$ is rationally impermissible to realize. And so, given this, there is good reason to think that we cannot extract deontic verdicts for the members of $\mathbf{F}^{I}$ from the deontic status of the members of $\mathbf{C}^{I}$.

The problem with maintaining that each of the members of $\mathbf{F}^{I}$ is rationally impermissible to realize, given that each of the members of $\mathbf{C}^{I}$ is rationally impermissible to realize, is that this commits us, in certain cases, to claiming that some $F \in \mathbf{F}^{I}$ is rationally impermissible, at least in part, in virtue of the fact that $i$ takes it to be sufficiently likely that were they to realize some coarse-grained credal option $C=F \cup \ldots$., they would not realize $F$, but would instead realize some other option that would result in lesser epistemic utility.

To see this, suppose, as the account under consideration maintains, that the members of $\mathbf{F}^{I}$ are rationally impermissible in virtue of the fact that each of the members of $\mathbf{C}^{I}$ is rationally permissible and the members of $\mathbf{F}^{I}$ are the fine-grainings of these impermissible coarse-grained options. Now, in standard cases, the members of $\mathbf{C}^{I}$ will be rationally impermissible in virtue of the fact that there is some set of options that maximize expected epistemic utility and the expected epistemic utility of the members of $\mathbf{C}^{I}$ are all less than this maximal value. But note that, amongst the fine-grainings of some $C \in \mathbf{C}^{I}$, there may be a credal option $F$ that does very well with respect to epistemic utility in various circumstances. Nonetheless, it may be that $C$ has low expected epistemic utility because the agent expects that were they to realize $C$ they would not realize $F$, but would instead realize some other option that results in greater epistemic utility. Since, then, $C$ has low expected utility in virtue of the fact that $i$ expects that were they to realize $C$ they would not realize $F$, and since $C$ is rationally impermissible, at least in part, in virtue of the fact that it has sufficiently low expected epistemic utility, if we, in addition, say that $F$ is rationally impermissible in virtue of the fact that $C$ is rationally impermissible, we seem to be forced to say that $F$ is rationally impermissible, at least in part, in virtue of the fact that $i$ expects that were they 
to realize $C$ they would not realize $F$, but would instead realize some epistemically inferior option.

I think, though, that taking it to be likely that were one to realize $C$ one would not realize $F$ but instead would realize some epistemically inferior option can't be the sort of fact that serves, even in part, to make the realization of $F$ rationally impermissible. Now this claim may be less obvious than the claim that taking it to be likely that were one to realize $C$ one would not realize $F$ but instead would realize some epistemically superior option can't be the sort of fact that serves, even in part, to make the realization of $F$ rationally permissible. But it seems to me that there is a common principle behind both of these claims that is simply more starkly illustrated in the latter case. For just as it seems plausible that an expectation that were one to realize some fine-grained option in some class, one would not realize some option $F$ but instead some other option with certain good-making features, can't itself be the sort of good-making feature that would make the realization of $F$ rationally permissible, so too should we think that an expectation that were one to realize some fine-grained option in some class, one would not realize some option $F$ but instead some other option with certain bad-making features, can't itself be the sort of bad-making feature that would make the realization of $F$ rationally impermissible. For, in neither case, does the fact in question seem to bear on $F$ in the right way to either rationally support or impugn its realization.

Given the preceding, then, we should conclude that we can't, in any plausible manner, extract verdicts about the deontic status of the members of $\mathbf{F}^{I}$, given the permissibility of the members of $\mathbf{C}^{I}$, or more generally, given the deontic status of the agent's coarse-grained options.

Since $\mathbf{F}^{P}$ and $\mathbf{F}^{I}$ collectively exhaust the fine-grained credal options available to an agent, and since in neither case can one extract verdicts about the deontic status of the members of these classes, given the deontic status of the agent's coarse-grained options, we should conclude that, in general, one cannot extract deontic verdicts about an agent's fine-grained credal options given deontic verdicts about the agent's coarse-grained credal options. If, then, one is a credal consequentialist and one takes $\mathbf{C}_{i}^{t^{\prime}}$ to be a set of unions of propositions uniquely characterizing $i$ 's possible credal distributions over $\mathbf{A}_{i}^{t^{\prime}}$, it seems to me that one must maintain that which particular credal state one has is not something that can be assessed as rationally permissible or impermissible.

This strikes me as a bad consequence. For, at least prima facie, it would seem that an adequate theory of credal rationality should provide deontic verdicts for the particular credal states that an agent may have and not merely for classes of such states.

While, then, a proponent of credal consequentialism can block the argument presented in $§ 2.2$ for the claim that, in a large class of cases, no deontic distinctions can be drawn amongst an agent's credal options, by taking $\mathbf{C}_{i}^{t^{\prime}}$ to be a set of unions of propositions uniquely characterizing $i$ 's possible credal distributions over $\mathbf{A}_{i}^{t^{\prime}}$, this move itself comes with significant costs. 


\subsection{Credal Quasi-Consequentialism}

I've argued that the proponent of credal consequentialism should endorse No Credal Distinctions, given that they endorse Credal Maximization. The argument for this claim took the following form. First, I argued that, given Maximization, one should endorse Global Dominance Permissivism, and that, given the latter principle, one should endorse No Distinctions. I then noted that these arguments may be repurposed, mutatis mutandis, to argue first that, given Credal Maximization, one should endorse Global Credal Dominance Permissivism, and second that, given the latter principle, one should endorse No Credal Distinctions.

Now, given our characterization of credal consequentialism, we should, I think, accept this argument. For, as I've understood it here, credal consequentialism is the view that, in general, the deontic status of an agent's credal options is determined by the degree to which the agent takes such options to be conducive to the epistemic good. Given this characterization, then, the proponent of credal consequentialism should take it that the principles governing credal rationality will be the same formally as the principles governing practical rationality, and will differ only materially with respect to the relevant notion of utility and the relevant options whose deontic status is under consideration.

It is worth noting, however, that there are other views of credal rationality that may have consequentialist elements, but that don't take all credal norms to follow from a more basic norm that enjoins an agent to try to adopt credences that have the best epistemic outcomes. And, given this sort of theory of credal rationality, one might in principle accept Credal Maximization while rejecting No Credal Distinctions.

For example, consider the following theory. On this view, there are two interacting components that determine the rationality of an agent's credal state, one consequentialist, one evidential. In those cases in which an agent has an appropriate expectation value for the epistemic utility of all of their possible credal states at some time $t^{\prime}$, an agent ought, according to this theory, to adopt a credal state that maximizes this expectation value (if there are such credal states). In those cases, though, in which an agent lacks appropriate expectation values for the epistemic utility of some of their possible credal states at $t^{\prime}$, then, according to this theory, the basic norms governing the agent's credences at $t^{\prime}$ are evidential. In particular, in such cases, an agent ought to have whatever credences are mandated by the agent's evidence.

On this two-tiered view, then, Credal Maximization holds, but if the agent lacks appropriate expectation values for the epistemic utility of an infinite number of credal states that they may adopt at $t^{\prime}$, it need not follow that no non-trivial deontic distinctions can be drawn for such credal states. For, in such cases, facts about the agent's evidence may serve to determine non-trivial deontic distinctions. This view, then, rejects No Credal Distinctions, while endorsing Credal Maximization.

Now I think it's far from obvious that this sort of two component theory is at all plausible. For present purposes, though, the point to note is simply that, while such views may endorse Credal Maximization and reject No Credal Distinctions, strictly speaking, they are not credal consequentialist views. For, on these views, not all norms follow from a basic norm enjoining pursuit of the epistemic good. 
A theory of credal rationality that appeals to consequentialist considerations, then, may avoid the arguments presented in this paper. Doing so, however, requires rejecting the ambitious idea that credal rationality can be understood fully in consequentialist terms.

\section{Conclusion}

When we look closely at the picture of rationality provided by credal consequentialism, the results appear to be very much at odds with our pre-theoretic judgments. Not only does this account, in certain circumstances, sanction probabilistic incoherence or the acceptance of epistemic bribes, but, given plausible assumptions, in a vast range of cases, this account fails to draw any interesting deontic distinctions. This makes for a very strange and implausible picture of credal rationality.

The problem stems from the fact that, given an account of credal rationality, the most natural bearers of deontic properties - namely, the particular credal states that an agent might adopt at some time - are, given the conceptual resources available to an agent who

might adopt such credal states, simply not the sorts of things about whose epistemic value an agent can, in general, have well-defined expectation values. This, I suggest, is a deep structural weakness with credal consequentialism.

\section{References}

Selim Berker. Epistemic teleology and the seperateness of propositions. The Philosophical Review, 122:337-393, 2013.

Michael Caie. Belief and indeterminacy. The Philosophical Review, 121(1):1-54, 2012.

Michael Caie. Rational probabilistic incoherence. The Philosophical Review, 122(4):527-575, 2013.

Michael Caie. Calibration and probabilism. Ergo, 1(1):13-38, 2014.

Michael Caie. Credence in the image of chance. Philosophy of Science, 82(4):626-648, 2015.

Catrin Campbell-Moore. Rational probabilistic incoherence? A reply to Michael Caie. The Philosophical Review, 124(3):393-406, 2015.

Jennifer Carr. Accuracy or coherence. MS, n.d.

David Chalmers. The Nature of Epistemic Space. In Andy Egan and Brian Weatherson, editors, Epistemic Modality. Oxford University Press, 2011.

Allan Gibbard and William Harper. Counterfactuals and two kinds of expected utility. In C. Leach, E. McClennen, and C. Hooker, editors, Foundations and Applications of Decision Theory, pages 125-162. Dordrecht: D. Reidel, 1978.

Jeremy Goodman. Consequences of conditional excluded middle. (MS), n.d. 
Hilary Greaves. Epistemic decision theory. Mind, 122(488):915-952, 2013.

Alan Hájek. A puzzle about degree of belief. (MS), n.d.

Thomas Jech. Set Theory. Springer Press, 2000.

C.S. Jenkins. Entitlement and rationality. Synthese, 157:25-45, 2007.

James Joyce. A non-pragmatic vindication of probabilism. Philosophy of Science, 65:575603, 1998.

James Joyce. The Foundations of Causal Decision Theory. Cambridge University Press, 1999.

James Joyce. Accuracy and coherence: Prospects for an alethic epistemology of partial belief. In F. Huber and C. Schmidt-Petri, editors, Degrees of Belief. Synthese Library, 2009.

Jason Konek and Ben Levinstein. The foundations of epistemic decision theory. Mind, forthcoming.

David Lewis. Counterfactuals. Harvard University Press, Cambridge, MA, 1973.

David Lewis. Counterfactual dependence and time's arrow. Nô̂s, 13(4):455-476, 1979a.

David Lewis. Attitudes de dicto and de se. The Philosophical Review, 88(4):513-543, 1979b.

David Lewis. Causal decision theory. Australasian Journal of Philosophy, 59:5-30, 1981.

Richard Pettigrew. Accuracy, chance, and the principal principle. The Philosophical Review, 121(2):241-275, 2012.

John L. Pollock. How do you maximize expectation value? Nô̂s, 17(3):409-421, 1983.

Robert Stalnaker. A defense of conditional excluded middle. In Ifs, pages 87-104. Springer, 1981a.

Robert Stalnaker. A theory of conditionals. In Ifs, pages 41-55. Springer, 1981b.

J.R.G. Williams. Defending conditional excluded middle. Noûs, 44(4):650-668, 2010. 